NBER WORKING PAPER SERIES

\title{
TRAINING, WAGES, AND SAMPLE SELECTION: ESTIMATING SHARP BOUNDS ON TREATMENT EFFECTS
}

\author{
David S. Lee \\ Working Paper 11721 \\ http://www.nber.org/papers/w11721 \\ NATIONAL BUREAU OF ECONOMIC RESEARCH \\ 1050 Massachusetts Avenue \\ Cambridge, MA 02138 \\ October 2005
}

Vivian Hwa provided excellent research assistance. I thank David Card, Guido Imbens, Justin McCrary, Enrico Moretti, and Jim Powell for helpful discussions and David Autor, Josh Angrist, John DiNardo, Jonah Gelbach, Alan Krueger, Doug Miller, Aviv Nevo, Jack Porter, Ed Vytlacil, Diane Whitmore, and participants of the UC Berkeley Econometrics and Labor Lunches, for useful comments and suggestions. The views expressed herein are those of the author(s) and do not necessarily reflect the views of the National Bureau of Economic Research.

(C2005 by David S. Lee. All rights reserved. Short sections of text, not to exceed two paragraphs, may be quoted without explicit permission provided that full credit, including (C) notice, is given to the source. 
Training, Wages, and Sample Selection: Estimating Sharp Bounds on Treatment Effects

David S. Lee

NBER Working Paper No. 11721

October 2005

JEL No. J0, J3, C1, C2, C5

\begin{abstract}
This paper empirically assesses the wage effects of the Job Corps program, one of the largest federally-funded job training programs in the United States. Even with the aid of a randomized experiment, the impact of a training program on wages is difficult to study because of sample selection, a pervasive problem in applied micro-econometric research. Wage rates are only observed for those who are employed, and employment status itself may be affected by the training program. This paper develops an intuitive trimming procedure for bounding average treatment effects in the presence of sample selection. In contrast to existing methods, the procedure requires neither exclusion restrictions nor a bounded support for the outcome of interest. Identification results, estimators, and their asymptotic distribution, are presented. The bounds suggest that the program raised wages, consistent with the notion that the Job Corps raises earnings by increasing human capital, rather than solely through encouraging work. The estimator is generally applicable to typical treatment evaluation problems in which there is non-random sample selection/attrition.

David S. Lee

Department of Economics

549 Evans Hall, \#3880

University of California

Berkeley, CA 94720-3880

and NBER

dslee@econ.berkeley.edu
\end{abstract}




\section{Introduction}

For decades, many countries around the world have administered government-sponsored employment and training programs, designed to help improve the labor market outcomes of the unemployed or economically disadvantaged. ${ }^{1}$ To do so, these programs offer a number of different services, ranging from basic classroom education and vocational training, to various forms of job search assistance. The key question of interest to policymakers is whether or not these programs are actually effective, sufficiently so to justify the cost to the public. The evaluation of these programs has been the focus of a large substantive and methodological literature in economics. Indeed, Heckman et al. [1999] observe that "[f]ew U.S. government programs have received such intensive scrutiny, and been subject to so many different types of evaluation methodologies, as governmentally-supplied job training."

Econometric evaluations of these programs typically focus on their reduced-from impacts on total earnings, a first-order issue for cost-benefit analysis. Unfortunately, exclusively studying the effect on total earnings leaves open the question of whether any earnings gains are achieved through raising individuals' wage rates (price effects) or hours of work (quantity effects). That is, a training program may lead to a meaningful increase in human capital, thus raising participants' wages. Alternatively, the program may have a pure labor supply effect: through career counseling and encouragement of individuals to enter the labor force, a training program may simply be raising incomes by increasing the likelihood of employment, without any increase in wage rates.

But assessing the impact of training programs on wage rates is not straightforward, due to the wellknown problem of sample selection, which is pervasive in applied micro-econometric research. That is, wages are only observed for individuals who are employed. Thus, even if there is random assignment of the "treatment" of a training program, there may not only be an effect on wages, but also on the probability that a person's wage will even be observed. Even a randomized experiment cannot guarantee that treatment and control individuals will be comparable conditional on being employed. Indeed, standard labor supply theory

\footnotetext{
${ }^{1}$ See Heckman, LaLonde and Smith [1999] for figures on expenditures on active labor market programs in OECD countries. See also Martin [2000].
} 
predicts that wages will be correlated with the likelihood of employment, resulting in sample selection bias [Heckman 1974]. This missing data problem is especially relevant for analyzing public job training programs, which typically target individuals who have low employment probabilities.

This paper empirically assesses the wage effects of the Job Corps program, one of the largest federallyfunded job training programs in the United States. ${ }^{2}$ The Job Corps is a comprehensive program for economically disadvantaged youth aged 16 to 24 , and is quite intensive: the typical participant will live at a local Job Corps center, receiving room, board, and health services while enrolled, for an average of about eight months. During the stay, the individual can expect to receive about 1100 hours of vocational and academic instruction, equivalent to about one year in high school. The Job Corps is also expensive: the average cost is about $\$ 14,000$ per participant. ${ }^{3}$ This paper uses data from the National Job Corps Study, a randomized evaluation funded by the U.S. Department of Labor.

Standard parametric or semi-parametric methods for correcting for sample selection require exclusion restrictions that have little justification in this case. As shown below, the data include numerous baseline variables, but all of those that are found be related to employment probabilities (i.e., sample selection) could also plausibly directly determine wage rates.

Thus, this paper develops an alternative method, a general procedure for bounding the treatment effects. The method amounts to first identifying the excess number of individuals who are induced to be selected (employed) because of the treatment, and then "trimming" the upper and lower tails of the outcome (e.g., wage) distribution by this number, yielding a worst-case scenario bound. The assumptions for identifying the bounds are already assumed in conventional models for sample selection: 1) the regressor of interest is independent of the errors in the outcome and selection equation, and 2) the selection equation can be written as a standard latent variable binary response model. In the case of an experiment, random

\footnotetext{
${ }^{2}$ In the 2004 fiscal year, the U.S. Department of Labor's Employment and Training Administration spent \$1.54 billion for the operation of the Job Corps. By comparison, it spent about $\$ 893$ million on "Adult Employment and Training Activities" (job search assistance for anyone and job training available to anyone if such training is needed for obtaining or retaining employment) and about $\$ 1.44$ billion on "Dislocated Workers Employment and Training Activities" (employment and training services for unemployment and underemployed workers) [U.S. Department of Labor 2005a].

3 A summary of services provided and costs can be found in Burghardt, Schochet, McConnell, Johnson, Gritz, Glazerman, Homrighausen and Jackson [2001].
} 
assignment ensures the first assumption holds. It is proven that the trimming procedure yields the tightest bounds for the average treatment effect that are consistent with the observed data. No exclusion restrictions are required and the bounds do not require a bounded support for the outcome variable.

An estimator for the bounds is introduced and shown to be $\sqrt{n}$ consistent and asymptotically normal with an intuitive expression for its asymptotic variance. It not only depends on the variance of the trimmed outcome variable, but also on the trimming threshold, which is an estimated quantile. There is also an added term that accounts for the estimation of which quantile (e.g., the 10th, 11th, 12th, etc. percentile) of the distribution to use as the trimming threshold.

For the analysis of Job Corps, the trimming procedure is instrumental to measuring the wage effects, producing bounds that are somewhat narrow. For example, at week 90 after random assignment, the estimated interval for the treatment effect is 4.2 to 4.3 percent, therefore ruling out a zero effect, even when wages are missing for about 54 percent of individuals. By the end of the 4-year follow-up period, the interval is still somewhat informative, more consistent with positive than negative effects, with an interval of -2 to 9 percent. By comparison, the assumption-free, "worst-case scenario" bounds proposed by Horowitz and Manski [2000a] produce a lower bound of -74 percent effect and an upper bound of 80 percent.

Overall, the evidence presented here points to a positive causal effect of the program on wage rates, although the magnitude probably does not exceed 10 percent. This is consistent with the view that the Job Corps program represents a human capital investment, rather than a means to improve earnings through raising work effort alone.

The proposed trimming procedure is neither specific to this application nor to randomized experiments. It will generally be applicable to treatment evaluation problems when outcomes are missing, a problem that often arises in applied research. Reasons for missing outcomes range from survey non-response (e.g., students not taking tests) to sample attrition (e.g., inability to follow individuals over time), to other structural reasons (e.g., mortality). Generally, this estimator is well-suited for cases where the researcher is uncomfortable imposing exclusion restrictions in the standard two-equation sample selection model, and when the support of the outcome variable is too wide to yield informative boudns on treatment effects. 
This paper is organized as follows. It begins, in Section II, with a description of the Job Corps program, the randomized experiment, and the nature of the sample selection problem. After this initial analysis, the proposed bounding procedure is described in Sections III and IV. Section III presents the identification results, while Section IV introduces a consistent and asymptotically normal estimator of the bounds, and discusses inference. Section V reports the results from the empirical analysis of the Job Corps. Section VI concludes.

\section{The National Job Corps Study and Sample Selection}

This section describes the Job Corps program and the data used for the analysis, replicates the main earnings results of the recently-completed randomized evaluation, and illustrates the nature of the sample selection problem. It is argued below that standard sample selection correction procedures are not appropriate for this context. Also, in order to provide an initial benchmark, the approach of Horowitz and Manski [2000a] is used to provide bounds on the Job Corps' effect on wages. They are to be compared to the "trimming" bounds presented in Section V, which implements the estimator developed in Sections III and IV.

\section{II.A. The Job Corps Program and the Randomized Experiment}

The U.S. Department of Labor describes the Job Corps program today as "a no-cost education and vocational training program ... that helps young people ages 16 through 24 get a better job, make more money and take control of their lives" [U.S. Department of Labor 2005b]. To be eligible, an individual must be a legal resident of the United States, be between the ages of 16 and 24, and come from a lowincome household. ${ }^{4}$ The administration of the Job Corps is considered to be somewhat uniform across the 110 local Job Corps centers in the United States.

Perhaps the most distinctive feature of the program is that most participants live at the local Job Corps center while enrolled. This residential component of the program includes formal social skills training, meals, and a dormitory-style life. During the stay, with the help of counselors, they develop individualized,

${ }^{4}$ Information on the Job Corps and the National Job Corps Study can be found in Schochet, Burghardt and Glazerman [2001]. 
self-paced programs which will consist of a combination of remedial high school education, including consumer and driver education, as well as vocational training in a number of areas, including clerical work, carpentry, automotive repair, building and apartment maintenance, and health related work. On average, enrollees can expect to receive about 440 hours of academic instruction and about 700 hours of vocational training, over an average of 30 weeks. Centers also provide health services, as well as job search assistance upon students' exit from the Job Corps.

In the mid-1990s, three decades after the creation of Job Corps, the U.S. Department of Labor funded a randomized evaluation of the program. ${ }^{5}$ Persons who applied for the program for the first time between November 1994 and December 1995, and were found to be eligible (80,883 persons) were randomized into a "program" and "control" group. The control group of 5977 individuals were essentially embargoed from the program for three years, while the remaining applicants could enroll in the Job Corps as usual. Since those who were still eligible after randomization were not compelled to participate, the differences in outcomes between program and control group members represents the reduced-form effect of eligibility, or the "intent-to-treat" effect. This treatment effect is the focus of the empirical analysis presented below. ${ }^{6}$

Of the program group, 9409 applicants were randomly selected to be followed for data collection. The research sample of 15386 individuals were interviewed at random assignment, and at three subsequent points in time, 12, 30, and 48 months after random assignment. Due to programmatic reasons, some subpopulations were randomized into the program group with differing, but known, probabilities. Thus, analyzing the data requires the use of the design-weights in the analysis. ${ }^{7}$

This paper uses the public-release data of the National Job Corps Study. Table I provides descriptive statistics for the data used in the analysis below. For baseline as well as post-assignment variables, it reports the treatment and control group means, standard deviations, proportion of the observations with non-missing values for the specified variable, as well as the difference in the means and associated standard error. The table shows that the proportion non-missing and the means for the demographic variables (the

\footnotetext{
5 The study was conducted by Mathematica Policy Research, Inc.

6 Throughout the paper, when I use the phrase "effect of the program", I am referring to this reduced-form treatment effect.

7 This paper uses the variable DSGN_WGT as described in Schochet, Cao, Glazerman, Grady, Gritz, McConnell, Johnson and Burghardt [2003].
} 
first 12 rows), education and background variables (the next 4 rows), income at baseline (the next 9 rows), and employment information (the next 6 rows) are quite similar. For only one of the variables - usual weekly hours on the most recent job at the baseline - is the difference ( 0.91 hours) statistically significant. A logit of the treatment indicator on all baseline characteristics in Table I was estimated; the chi-square test of the coefficients all being zero yielded a p-value of $0.577 .^{8}$ The overall comparability between the treatment and control groups is consistent with successful randomization of the treatment.

It is important to note that the analysis in this paper abstracts from missing values due to interview non-response and sample attrition over time. Thus, only individuals who had non-missing values for weekly earnings and weekly hours for every week after the random assignment are used; the estimation sample is thus somewhat smaller (9145 vs. 15386). It will become clear below that the trimming procedure could be applied exclusively to the attrition/non-response problem, which is a mechanism for sample selection that is quite distinct from the selection into employment status. More intensive data collection can solve the attrition/non-response problem, but not the sample selection on wages caused by employment. For this reason, the analysis below focuses exclusively on the latter problem, and analyzes the data conditional on having continuously valid earnings and hours data. ${ }^{9}$

The bottom of Table I shows that the only set of variables that show important (and statistically significant) differences between treatment and control are the post-assignment labor market outcomes. The treatment group has lower weekly hours and earnings at week 52, but higher hours and earnings at the 3-year and 4-year marks. At week 208, the earnings gain is about 27 dollars, with the control mean of about 200 dollars. The effect on weekly hours at that time is a statistically significant 1.95 hours. ${ }^{10}$

\footnotetext{
${ }^{8}$ Missing values for each of the baseline variables were imputed with the mean of the variable. The analysis below uses this imputed data.

9 Although the analysis here abstracts from the non-response problem, there is some evidence that it is a second-order issue. The proportion of control group individuals, at week 90, that have continuously non-missing earnings and hours data is 0.822 , and the proportion is 0.003 smaller (standard error of 0.006 ) for the treatment group. If the analysis below is applied to the attrition problem, it implies that there is no attrition bias. An analogous calculation for any week from the 48-month interview (including week 208) will necessarily not yield the same zero effect. This is because, by design, fewer treatment group individuals were contacted, due to data collection costs. Mathematica Policy Research, Inc. "randomly selected for 48-month interviewing 93 percent of program group members who were eligible for 48-month interviews" [Schochet et al. 2003].

10 This is consistent with Mathematica's final report, which showed that the program had about a 12 percent positive effect on earnings by the fourth year after enrollment, and suggested that lifetime gains in earnings could very well exceed the program's costs [Burghardt et al. 2001].
} 
Figure I illustrates the treatment effects on earnings for each week subsequent to random assignment. It shows an initial negative impact on earnings for the first 80 weeks, after which point a positive treatment effect appears and grows. The estimates in the bottom of Table I and plotted in Figure I are similar qualitatively and quantitatively to the impact estimates reported in Schochet et al. [2001]. ${ }^{11}$

\section{II.B. The Effect on Wages and the Sample Selection Problem}

It seems useful to assess the impact of the program on wage rates, as distinct from total earnings - which is a product of both the price of labor (the wage) and labor supply (whether the person works, and if so, how many hours). Distinguishing between price and quantity effects is important for better understanding the mechanism through which Job Corps leads to more favorable labor market outcomes.

On the one hand, one of the goals of the Job Corps is to encourage work and self-sufficiency; thus, participants' total earnings might rise simply because the program succeeds in raising the likelihood that they will be employed, while at the same time leaving the market wage for their labor unaffected. On the other hand, the main component of the Job Corps is significant academic and vocational training, which could be expected to raise wages. There is a great deal of empirical evidence to suggest a positive causal effect of education on wages. ${ }^{12}$

Unfortunately, even though the National Job Corps study was a randomized experiment, one cannot use simple treatment-control differences to estimate the effect of the program on wage rates. This is because the effective "prices" of labor for these individuals are only observed to the econometrician when the individuals are employed. This gives rise to the classic sample selection problem (e.g., see Heckman [1979]).

Figure II suggests that sample selection may well be a problem for the analysis of wage effects of the Job Corps. It reports employment rates (the proportion of the sample that has positive work hours in the week) for both treated and control individuals, for each week following random assignment. The results

\footnotetext{
11 In Schochet et al. [2001], the reported estimates used a less stringent sample criterion. Instead of requiring non-missing values for 208 consecutive weeks, individuals only needed to complete the 48-month interview (11313 individuals). Therefore, for that sample, some weeks' data will be missing. Despite the difference in the samples, both the levels, impact estimates, and time profile reported in Schochet et al. [2001] are also quite similar to those found in Figures II, and III (below).

12 For a survey of the recent literature on the causal effect of education on earnings, see Card [1999].
} 
show that the program had a negative impact on employment propensities in the first half of the follow-up, and a positive effect in the latter half. This shows that the Job Corps itself affected whether individuals would have a non-missing wage rate.

Put another way, Figure II illustrates that even though proper random assignment will imply treated and control groups are comparable at the baseline, they may well be systematically different conditional on being employed in a given period subsequent to the random assignment. As a result, the treatment-control difference in mean log-hourly wages, as plotted in Figure III, may not represent the true causal effect of the program. ${ }^{13}$

There are two other reasons why sample selection can potentially be important in this case. As shown in Figure II, a large fraction of individuals are not employed: employment rates start at about 20 percent and grow to at most 60 percent at the four-year mark. Second, non-employed and employed individuals appear to be systematically different on a number of important observable dimensions. Table II reports log-odds coefficients from a logit of employment in week 208 on the treatment dummy and the baseline characteristics listed in Table I. As might be expected, gender, race, education, criminal history, and employment status at the baseline are all very strong predictors of employment in week 208.

The problem of non-random sample selection is well understood in the training literature; it may be one of the reasons why most evaluations of job training programs focus on total earnings, including zeros for those without a job, rather than on wages conditional on employment. Of the 24 studies referenced in a survey of experimental and non-experimental studies of U.S. employment and training programs [Heckman et al. 1999], most examine annual, quarterly, or monthly earnings without discussing the sample selection problem of examining wage rates. ${ }^{14}$ As for the Job Corps, when reporting results on hourly wages for the working, Schochet et al. [2001] is careful to note that because of the selection into employment, the

\footnotetext{
13 Hourly wage is computed by dividing weekly earnings by weekly hours worked, for the treatment and control group. Note the pattern of "kinks" that occur at the 12- and 30-month marks, which is also apparent in Figure I. This could be caused by the retrospective nature of the interviews that occur at 12-, 30-, and 48-months post-random-assignment. This pattern would be found if there were systematic over-estimation of earnings on employment that was further away from the interview date. The lines would "connect" if respondents were reminded of their answer from the previous interview. Note that these potential errors do not seem to be too different between the treatment and control groups, as there are no obvious kinks in the difference (solid squares).

14 The exceptions include Kiefer [1979], Hollister, Kemper and Maynard [1984], and Barnow [1987]. The sources from Tables 22 and 24 in Heckman et al. [1999] were surveyed.
} 
treatment-control differences cannot be interpreted as impact estimates.

\section{II.C. Existing Approaches}

Currently, there are two general approaches to addressing the sample selection problem. The first is to explicitly model the process determining selection. The conventional setup, following Heckman [1979], models the wage determining process as

$$
\begin{aligned}
Y^{*} & =D \beta+X \pi_{1}+U \\
Z^{*} & =D \gamma+X \pi_{2}+V \\
Y & =1\left[Z^{*} \geq 0\right] \cdot Y^{*}
\end{aligned}
$$

where $Y^{*}$ is the offered market wage as of a particular point in time (e.g., week 208 after randomization), $D$ is the indicator variable of receiving the treatment of being given access to the Job Corps program, and $X$ is a vector of baseline characteristics. $Z^{*}$ is a latent variable representing the propensity to be employed. $\gamma$ represents the causal effect of the treatment on employment propensities, while $\beta$ is the causal parameter of interest. $^{15}$

Both $Y^{*}$ and $Z^{*}$ are unobserved, but the wage conditional on employment $Y$ is observed, where $1[\cdot]$ is the indicator variable. $(U, V)$ are assumed to be jointly independent of the regressors $(D, X) .{ }^{16}$ As in Heckman [1979], sample selection bias can be seen as specification error in the conditional expectation

$$
E\left[Y \mid D, X, Z^{*} \geq 0\right]=D \beta+X \pi_{1}+E\left[U \mid D, X, V \geq-D \gamma-X \pi_{2}\right]
$$

One modeling approach is to assume that data are missing at random, perhaps conditional on a set of covariates [Rubin 1976]. This amounts to assuming that $U$ and $V$ are independent of one another, or that employment status is unrelated to the determination of wages. This assumption is strictly inconsistent with standard models of labor supply that account for the participation decision (e.g., see Heckman [1974]).

A more common modeling assumption is that some of the exogenous variables determine sample selection, but do not have its own direct impact on the outcome of interest; that is, some of the elements

\footnotetext{
$\overline{15}$ In this specification, the treatment effect is constant.

16 This assumption, which is stronger than necessary, is invoked now for expositional purposes. It will be shown below that what is required is instead independence of $(U, V)$ and $D$, conditional on $X$.
} 
of $\pi_{1}$ are zero while corresponding elements of $\pi_{2}$ are nonzero). Such exclusion restrictions are utilized in parametric and semi-parametric models of the censored selection process (e.g., Heckman [1979], Heckman [1990], Ahn and Powell [1993], Andrews and Schafgans [1998], and Das, Newey and Vella [2003]).

The practical limitation to relying on exclusion restrictions for the sample selection problem is that there may not exist credible "instruments" that can be excluded from the outcome equation. This seems to be true for an analysis of the Job Corps experiment. There are many variables available to the researcher from the Job Corps evaluation, and many of the key variables are listed in Tables I and II. But for each of the variables in Table II that have significant associations with employment, there is a well-developed literature suggesting that those variables may also influence wage offers. For example, race, gender, education, and criminal histories all could potentially impact wages. Household income and past employment experiences are also likely to be correlated with unobserved determinants of wages.

Researchers' reluctance to rely upon specific exclusion restrictions motivates a second, general approach to addressing the sample selection problem: the construction of "worst-case" bounds of the treatment effect. When the support of the outcome is bounded, the idea is to impute the missing data with either the largest or smallest possible values to compute the largest and smallest possible treatment effects consistent with the data that is observed. Horowitz and Manski [2000a] use this notion to provide a general framework for constructing bounds for treatment effect parameters when outcome and covariate data are non-randomly missing in an experimental setting. ${ }^{17}$ This strategy is discussed in detail in Horowitz and Manski [2000a], who show the approach can be useful when $Y$ is a binary outcome.

This imputation procedure cannot be used when the support is unbounded. Even when the support is bounded, if it is very wide, so too will the width of the treatment effect bounds. In the context of the Job Corps program, the bounds are somewhat uninformative. Table III computes the Horowitz and Manski [2000a] bounds for the treatment effect of the Job Corps program on log-wages in week 208. Specifically,

\footnotetext{
17 Others [Balke and Pearl 1997, Heckman and Vytlacil 1999, Heckman and Vytlacil 2000b, Heckman and Vytlacil 2000a] have constructed such bounds to address a very different problem - that of imperfect compliance of the treatment, even when "intention" to treat is effectively randomized [Bloom 1984, Robins 1989, Imbens and Angrist 1994, Angrist, Imbens and Rubin 1996].
} 
it calculates the upper bound of the treatment effect as

$$
\begin{aligned}
& \operatorname{Pr}\left[Z^{*} \geq 0 \mid D=1\right] E[Y \mid D=1]+\operatorname{Pr}\left[Z^{*}<0 \mid D=1\right] Y^{U B} \\
& -\operatorname{Pr}\left[Z^{*} \geq 0 \mid D=0\right] E[Y \mid D=0]+\operatorname{Pr}\left[Z^{*}<0 \mid D=0\right] Y^{L B}
\end{aligned}
$$

where all population quantities can be estimated, and $Y^{U B}$ and $Y^{L B}$ are the upper and lower bounds of the support of log-wages; as reported in the Table, $Y^{U B}$ and $Y^{L B}$ are taken to be 2.77 and 0.90 (\$15.96 and $\$ 2.46$ an hour), respectively. ${ }^{18}$

Table III shows that the lower bound for the treatment effect on week 208 log-wages is -0.75 and the upper bound is 0.80 . Thus, the interval is almost as consistent with extremely large negative effects as it is with extremely large positive effects. The reason for this wide interval is that more than 40 percent of the individuals are not employed in week 208. In this context, imputing the missing values with the maximal and minimal values of $Y$ is so extreme as to yield an interval that includes effect sizes that are arguably implausible. Nevertheless, the Horowitz and Manski [2000a] bounds provide a useful benchmark, and highlights that some restrictions on the sample selection process are needed to produce tighter bounds [Horowitz and Manski 2000b].

The procedure proposed below is a kind of "hybrid" of the two general approaches to the sample selection problem. It yields bounds on the treatment effect, even when the outcome is unbounded. It does so by imposing some structure on the sample selection process, but without requiring exclusion restrictions.

\section{Identification of Bounds on Treatment Effects}

This section first uses a simple case in order to illustrate the intuition behind the main identification result, and then generalizes it for a very unrestrictive sample selection model.

Consider the case where there is only the treatment indicator, with no other covariates. That is, $X$ is a constant, so that $\pi_{1}$ and $\pi_{2}$ will be intercept terms. It will become clear that the result below is also

\footnotetext{
18 The wage variable was transformed before the being analyzed, in order to minimize the effect of outliers, and also so that the Horowitz and Manski [2000a] bounds would not have to rely on these outliers. Specifically, the entire observed wage distristribution was split into 20 categories, according to the 5 th, 10 th, 15 th,$\ldots 95$ th percentile wages, and the individual was assigned the mean wage within each of the 20 groups. Thus, the upper "bound" of the support, for example, is really the mean log-wage for those earning more than the 95 th percentile. The same data are used for the trimming procedure described below.
} 
valid conditional for any value of $X$. Describing the identification result in this simple case makes clear that the proposed procedure does not rely on exclusion restrictions. In addition, this section and the next assumes that $U$ (and hence $Y$ ) has a continuous distribution. Doing so will simplify the exposition; it can be shown that the proposed procedure can be applied to discrete outcome variables as well. ${ }^{19}$ Without loss of generality, assume that $\gamma>0$, so that the treatment causes an increase in the likelihood of the outcome being observed.

From Equation (1), the observed population means for the control and treatment groups can be written as

$$
E\left[Y \mid D=0, Z^{*} \geq 0\right]=\pi_{1}+E\left[U \mid D=0, V \geq-\pi_{2}\right]
$$

and

$$
E\left[Y \mid D=1, Z^{*} \geq 0\right]=\pi_{1}+\beta+E\left[U \mid D=1, V \geq-\pi_{2}-\gamma\right]
$$

This shows that when $U$ and $V$ are correlated, the difference in the means will generally be biased for $\beta$.

Identification of $\beta$ would be possible if we could estimate

$$
E\left[Y \mid D=1, V \geq-\pi_{2}\right]=\pi_{1}+\beta+E\left[U \mid D=1, V \geq-\pi_{2}\right]
$$

because (2) could be subtracted to yield the effect $\beta$ (since $D$ is independent of $(U, V)$ ). But the mean in (4) is not observed.

It can be bounded, however. This is because all observations on $Y$ needed to compute this mean are a subset of the selected population $\left(V \geq-\pi_{2}-\gamma\right)$. For example, we know that

$$
E\left[Y \mid D=1, Z^{*} \geq 0\right]=(1-p) E\left[Y \mid D=1, V \geq-\pi_{2}\right]+p E\left[Y \mid D=1,-\pi_{2}-\gamma \leq V<-\pi_{2}\right]
$$

where $p=\frac{\operatorname{Pr}\left[-\pi_{2}-\gamma \leq V<-\pi_{2}\right]}{\operatorname{Pr}\left[-\pi_{2}-\gamma \leq V\right]}$. The observed treatment mean is a weighted average of (4) and the mean for a sub-population of "marginal" individuals $\left(-\pi_{2}-\gamma \leq V<-\pi_{2}\right)$ who are induced to be selected into the sample because of the treatment.

$E\left[Y \mid D=1, V \geq-\pi_{2}\right]$ is therefore bounded above by $E\left[Y \mid D=1, Z^{*} \geq 0, Y \geq y_{p}\right]$, where $y_{p}$ is the $p$ th quantile of the treatment group's observed $Y$ distribution. This is true because among the selected

\footnotetext{
19 See Lee [2002], for an implementation of the bounds for a binary response outcome.
} 
population $V \geq-\pi_{2}-\gamma, D=1$, no sub-population with proportion $(1-p)$ can have a mean that is larger than the average of the largest $(1-p)$ values of $Y$.

Put another way, we cannot identify which observations are inframarginal $\left(V \geq-\pi_{2}\right)$ and which are marginal $\left(-\pi_{2}-\gamma \leq V<-\pi_{2}\right)$. But the "worst-case" scenario is that the smallest $p$ values of $Y$ belong to the marginal group and the largest $1-p$ values belong to the inframarginal group. Thus, by trimming the lower tail of the $Y$ distribution by the proportion $p$, we obtain an upper bound for the inframarginal group's mean in (4). Consequently, $E\left[Y \mid D=1, Z^{*} \geq 0, Y \geq y_{p}\right]-E\left[Y \mid D=0, Z^{*} \geq 0\right]$ is an upper bound for $\beta$. Note that the trimming proportion $p$ is equal to

$$
\frac{\operatorname{Pr}\left[Z^{*} \geq 0 \mid D=1\right]-\operatorname{Pr}\left[Z^{*} \geq 0 \mid D=0\right]}{\operatorname{Pr}\left[Z^{*} \geq 0 \mid D=1\right]}
$$

where each of these probabilities is identified by the data.

To summarize, a standard latent-variable sample selection model implies that the observed outcome distribution for the treatment group is a mixture of two distributions: 1) the distribution for those who would have been selected irrespective of the treatment (the inframarginal group), and 2) the distribution for those induced into being selected because of the treatment (the marginal group). It is possible to quantify the proportion of the treatment group that belongs to this second group, using a simple comparison of the selection probabilities of the treatment and control groups. Although it is impossible to identify specifically which treated individuals belong to the second group, worst-case scenarios can be constructed by assuming that they are either at the very top or the very bottom of the distribution. Thus, trimming the data by the known proportion of excess individuals should yield bounds on the mean for the inframarginal group.

\section{III.A. Identification under a Generalized Sample Selection Model}

This identification result applies to a much wider class of sample selection models. It depends neither on a constant treatment effect, nor on homoskedasticity, which are both implicitly assumed in Equation (1).

To see this, consider a general sample selection model that allows for heterogeneity in treatment ef- 
fects:

$$
\begin{aligned}
& \left(Y_{1}^{*}, Y_{0}^{*}, S_{1}, S_{0}, D\right) \text { is i.i.d. across individuals } \\
S= & S_{1} D+S_{0}(1-D) \\
Y= & S \cdot\left\{Y_{1}^{*} D+Y_{0}^{*}(1-D)\right\} \\
& (Y, S, D) \text { is observed }
\end{aligned}
$$

where $D, S, S_{0}$, and $S_{1}$ are all binary indicator variables. $D$ denotes treatment status; $S_{1}$ and $S_{0}$ are "potential" sample selection indicators for the treated and control states. For example, when an individual has $S_{1}=1$ and $S_{0}=0$, this means the outcome $Y$ will be observed $(S=1)$ if treatment is given, and will not be observed $(S=0)$ if treatment is denied. The second line highlights the fact that for each individual we only observe $S_{1}$ or $S_{0} . Y_{1}^{*}$ and $Y_{0}^{*}$ are latent potential outcomes for the treated and control states, and the third line points out we observe either latent outcome $Y_{1}^{*}$ or $Y_{0}^{*}$, and only if the individual is selected into the sample $S=1$. It is assumed throughout that $E[S \mid D=1], E[S \mid D=0]>0$.

Assumption 1 (Independence): $\left(Y_{1}^{*}, Y_{0}^{*}, S_{1}, S_{0}\right)$ is independent of $D$.

This assumption corresponds to the independence of $(U, V)$ and $(D, X)$ in the previous section. In the context of experiments, random assignment will ensure this assumption will hold.

Assumption 2 (Monotonicity): Either $S_{1} \geq S_{0}$ with probability 1, or $S_{0} \geq S_{1}$ with probability 1 .

This assumption implies that treatment assignment can only affect sample selection in "one direction". Some individuals will never be observed, regardless of treatment assignment ( $\left.S_{0}=S_{1}=0\right)$, others will always be observed ( $\left.S_{0}=1, S_{1}=1\right)$, and others will be selected into the sample because of the treatment $\left(S_{0}=0, S_{1}=1\right)$. This assumption is commonly invoked in studies of imperfect compliance of treatment [Imbens and Angrist 1994, Angrist et al. 1996]; the difference is that in those studies, monotonicity is for how an instrument affects treatment status; here, the monotonicity is for how treatment effects sample 
selection. It should be noted that monotonicity has been shown to be equivalent to assuming a latentvariable threshold-crossing model [Vytlacil 2002], which is the basis for virtually all sample selection models in econometrics.

Proposition 1: Let $Y_{0}^{*}$ and $Y_{1}^{*}$ be continuous random variables. If Assumptions 1 and 2 hold, and without loss of generality let $S_{1} \geq S_{0}$ with probability 1 , then $\Delta_{0}^{L B}$ and $\Delta_{0}^{U B}$ are sharp lower and upper bounds for the average treatment effect $E\left[Y_{1}^{*}-Y_{0}^{*} \mid S_{0}=1, S_{1}=1\right]$, where

$$
\begin{aligned}
\Delta_{0}^{L B} & \equiv E\left[Y \mid D=1, S=1, Y \leq y_{1-p_{0}}\right]-E[Y \mid D=0, S=1] \\
\Delta_{0}^{U B} & \equiv E\left[Y \mid D=1, S=1, Y \geq y_{p_{0}}\right]-E[Y \mid D=0, S=1] \\
y_{q} & \equiv G^{-1}(q), \text { with } G \text { the cdf of } Y, \text { conditional on } D=1, S=1 \\
p_{0} & \equiv \frac{\operatorname{Pr}[S=1 \mid D=1]-\operatorname{Pr}[S=1 \mid D=0]}{\operatorname{Pr}[S=1 \mid D=1]}
\end{aligned}
$$

The bounds are sharp in the sense that $\Delta_{0}^{L B}\left(\Delta_{0}^{U B}\right)$ is the largest (smallest) lower (upper) bound that is consistent with the observed data. ${ }^{20}$

Remark 1. The sharpness of the bound $\Delta_{0}^{U B}$, for example, means that it is the "best" upper bound that is consistent with the data. A specific example of where this proposition can be applied is in Krueger and Whitmore [2001], who study the impact of the Tennessee STAR class-size experiment. In that study, students are randomly assigned to a regular or a small class and the outcome of interest is the SAT (or ACT) scores, but not all students take the exam. On p. 25, Krueger and Whitmore [2001] utilize Assumptions 1 and 2 to derive a different upper bound, given by $B \equiv E[Y \mid D=1, S=1] \cdot \frac{\operatorname{Pr}[S=1 \mid D=1]}{\operatorname{Pr}[S=1 \mid D=0]}-E[Y \mid$ $D=0, S=1]$. Proposition 1 implies that this bound $B$, like any other proposed bound utilizing these assumptions, cannot be smaller than $\Delta_{0}^{U B} \cdot{ }^{21}$

Remark 2. An important practical implication of Assumptions 1 and 2 is that as $p_{0}$ vanishes, so does the sample selection bias. ${ }^{22}$ The intuition is that if $p_{0}=0$, then under the monotonicity assumption, both treatment and control groups are comprised of individuals whose sample selection was unaffected

\footnotetext{
${ }^{20}$ If $S_{0} \geq S_{1}$ with probability 1 , then the control group's, rather than the treatment group's, outcome distribution must be trimmed.

21 Thus, in the context of Krueger and Whitmore [2001], Proposition 1 implies that computing the bound $B$ is unncessary after already computing a very different estimate $T$, their "linear truncation" estimate. They justify $T$ under a different set of assumptions: 1) that "the additional small-class students induced to take the ACT exam are from the left tail of the distribution" and 2) "if attending a small class did not change the ranking of students in small classes." Their estimate $T$ is mechanically equivalent to the bound $\Delta_{0}^{U B}$. Therefore, Proposition 1 implies that their estimate $T$ is actually the sharp upper bound given the mild assumptions that were used to justify their bound $B$.

22 A vanishing $p$ corresponds to individuals with the same value of the sample selection correction term, and it is well known that there is no selection bias, conditional on the correction term. See, for example, Heckman and Robb [1986], Heckman [1990], Ahn and Powell [1993], and Angrist [1997].
} 
by the assignment to treatment, and therefore the two groups are comparable ${ }^{23}$. Thus, when analyzing randomized experiments, if the sample selection rates in the treatment and control groups are similar, and if the monotonicity condition is believed to hold, then a comparison of the treatment and control means is a valid estimate of an average treatment effect. ${ }^{24}$

Remark 3. Assumptions 1 and 2 are minimally sufficient for computing the bounds. Monotonicity ensures that the sample-selected control group consists only of those individuals with $S_{0}=1, S_{1}=1$. Without monotonicity, the control group could consist solely of observations with $S_{0}=1, S_{1}=0$, and the treatment group solely of observations with $S_{0}=0, S_{1}=1$. Since the two sub-populations do not "overlap", the difference in the means could not be interpreted as a causal effect. The independence assumption is also important, since it is what justifies the contrast between the trimmed treatment group and the control group.

Remark 4. When $p_{0}=0$ in a randomized experimental setting, there is a limited test of whether the simple difference in means suffers from sample selection bias. Suppose that each of the four subpopulations, defined by $\left(S_{0}=0, S_{1}=1\right),\left(S_{0}=1, S_{1}=0\right),\left(S_{0}=0, S_{1}=0\right)$, or $\left(S_{0}=1, S_{1}=1\right)$, have a different distribution of baseline characteristics $X$. If $p_{0}=0$ and monotonicity holds, then both treatment groups will consist solely of the $\left(S_{0}=1, S_{1}=1\right)$ group; thus, the of $X \mathrm{~s}$ should be the same in the treated and control groups, conditional on being selected. If monotonicity does not hold, then the selected, treated group will comprise of two sub-populations, $\left(S_{0}=1, S_{1}=1\right)$ and $\left(S_{0}=0, S_{1}=1\right)$, while the control group will be comprised of the groups $\left(S_{0}=1, S_{1}=1\right)$ and $\left(S_{0}=1, S_{1}=0\right)$, which predicts that there should be treatment-control differences in the distribution of $X \mathrm{~s}$, conditional on being selected.

Finally, the trimming procedure described above places sharp bounds on the average treatment effect for a particular sub-population - those individuals who will be selected irrespective of the treatment assignment $\left(S_{0}=0, S_{1}=1\right)$. It should be noted, however, that this sub-population is the only one for which

\footnotetext{
23 These individuals can be thought of as the "always-takers" sub-population [Angrist et al. 1996], except that "taking" is not the taking of the treatment, but rather selection into the sample.

24 Note that $p_{0}$ here is proportional to the difference in the fraction that are sample selected between the treatment and control groups. Thus, the notion of a vanishing $p$ should not be confused with "identification at infinity" in Heckman [1990], in which the bias term vanishes as the fraction that is selected into the sample tends to 1 .
} 
it is possible to learn about treatment effects, given Assumptions 1 and 2 (at least, in this missing data problem). For the marginal ( $S_{0}=0, S_{1}=1$ ) observations, the outcomes are missing in the control regime. For the remaining ( $S_{0}=0, S_{1}=0$ ) observations, outcomes are missing in both the treatment and control regimes. It would still be possible to appeal to the bounds of Horowitz and Manski [2000a] to construct bounds on this remaining population of the "never observed", but this interval (whose width would be 2 times the width of the outcome variable's support) would not require any data. Whether the sub-population of the "always observed" is of interest will depend on the context. In the case of the Job Corps program, for example, it is useful to assess the impact of the program on wage rates for those whose employment status was not affected by the program.

\section{Estimation and Inference}

This section proposes and discusses an estimator for the bounds. The estimator can be shown to be $\sqrt{n}$ consistent and asymptotically normal. The asymptotic variance is comprised of three components, reflecting 1) the variance of the trimmed distribution, 2) the variance of the estimated trimming threshold, and 3) the variance in the estimate of how much of the distribution to trim. To minimize redundancies, the discussion below continues to consider the case that $S_{1} \geq S_{0}$ with probability 1 (from Assumption 2); the results are also analogously valid for the reverse case of $S_{0} \geq S_{1}$.

\section{IV.A. Estimation}

The estimates of the bounds are sample analogs to the parameters defined in Proposition 1. First, the trimming proportion $\widehat{p}$ is estimated by taking the treatment-control difference in the proportion with nonmissing outcomes, and dividing by the proportion that is selected in the treatment group. Next, the $\widehat{p}$ th (or the $(1-\widehat{p})$ th) quantile of the treatment group's outcome distribution is calculated. Finally, these quantiles

are used to trim the data for the treatment group's outcomes and compute the bounds $\widehat{\Delta^{L B}}$ and $\widehat{\Delta^{U B}}$.

Formally, we have 


\section{Definition of Estimator.}

$$
\begin{aligned}
\widehat{\Delta^{L B}} & \equiv \frac{\sum Y \cdot S \cdot D \cdot 1\left[Y \leq \widehat{y_{1-\widehat{p}}}\right]}{\sum S \cdot D \cdot 1\left[Y \leq \widehat{y_{1-\widehat{p}}}\right]}-\frac{\sum Y \cdot S \cdot(1-D)}{\sum S \cdot(1-D)} \\
\widehat{\Delta^{U B}} & \equiv \frac{\sum Y \cdot S \cdot D \cdot 1\left[Y \geq \widehat{y_{\widehat{p}}}\right]}{\sum S \cdot D \cdot 1\left[Y \geq \widehat{y_{\widehat{p}}}\right]}-\frac{\sum Y \cdot S \cdot(1-D)}{\sum S \cdot(1-D)} \\
\widehat{y_{q}} & \equiv \min \left\{y: \frac{\sum S \cdot D \cdot 1[Y \leq y]}{\sum S \cdot D} \geq q\right\} \\
\widehat{p} & \equiv\left(\frac{\sum S \cdot D}{\sum D}-\frac{\sum S \cdot(1-D)}{\sum(1-D)}\right) /\left(\frac{\sum S \cdot D}{\sum D}\right)
\end{aligned}
$$

where the summation is over the entire sample of size $n$.

\section{IV.B. Consistency, Asymptotic Normality, Variance Estimation, and Inference}

The estimators $\widehat{\Delta^{L B}}$ and $\widehat{\Delta^{U B}}$ are consistent for $\Delta_{0}^{L B}$ and $\Delta_{0}^{U B}$ under fairly standard conditions:

Proposition 2 (Consistency): Let $\Delta_{0}^{L B}, \Delta_{0}^{U B} \in \boldsymbol{\Delta}$, which is compact, and $E[|Y|]<\infty$. Then $\widehat{\Delta^{L B}} \stackrel{p}{\rightarrow} \Delta_{0}^{L B}$ and $\widehat{\Delta^{U B}} \stackrel{p}{\rightarrow} \Delta_{0}^{U B}$

As shown in the Appendix, the proof involves showing that the estimator is a solution to a GMM problem, showing that the moment function vector is, with probability 1 , continuous at each $\Delta_{0}^{L B}, \Delta_{0}^{U B} \in$ $\boldsymbol{\Delta}$, and applying Theorem 2.6 of Newey and McFadden [1994].

The estimators $\widehat{\Delta^{L B}}$ and $\widehat{\Delta^{U B}}$ are also asymptotically normal, with an intuitive expression for the variance.

Proposition 3 (Asymptotic Normality): Define $\mu^{L B} \equiv E\left[Y \mid D=1, S=1, Y \leq y_{1-p_{0}}\right]$ and $\mu^{U B} \equiv E\left[Y \mid D=1, S=1, Y \geq y_{p_{0}}\right]$. In addition to the conditions in Proposition 2, let $\Delta_{0}^{L B}, \Delta_{0}^{U B}$ be interior points in $\Delta$, and let $E|Y|^{2+\delta}$ for some $\delta>0$. Then $\sqrt{n}\left(\widehat{\Delta^{L B}}-\Delta_{0}^{L B}\right) \stackrel{d}{\rightarrow}$ $N\left(0, V^{L B}+V_{C}\right)$ and $\sqrt{n}\left(\widehat{\Delta^{U B}}-\Delta_{0}^{U B}\right) \stackrel{d}{\rightarrow} N\left(0, V^{U B}+V_{C}\right)$, where

$$
\begin{aligned}
& V^{L B}=\frac{1}{E[S D]\left(1-p_{0}\right)}\left\{\operatorname{Var}\left[Y \mid D=1, S=1, Y \leq y_{1-p_{0}}\right]+\left(y_{1-p_{0}}-\mu^{L B}\right)^{2} p_{0}\right\} \\
& +\left(y_{1-p_{0}}-\mu^{L B}\right)^{2}\left(\frac{(1-E[S \mid D=0])-p_{0}(1-E[D])}{E[D] \cdot E[S \mid D=0] \cdot(1-E[D])}\right) \\
& V^{U B}=\frac{1}{E[S D]\left(1-p_{0}\right)}\left\{\operatorname{Var}\left[Y \mid D=1, S=1, Y \geq y_{p_{0}}\right]+\left(y_{p_{0}}-\mu^{U B}\right)^{2} p_{0}\right\} \\
& +\left(y_{p_{0}}-\mu^{U B}\right)^{2}\left(\frac{(1-E[S \mid D=0])-p_{0}(1-E[D])}{E[D] \cdot E[S \mid D=0] \cdot(1-E[D])}\right)
\end{aligned}
$$

and $V_{C}$ is the usual asymptotic variance of the estimated mean for the control group (divided by 


$$
E[S(1-D)])^{25}
$$

Consider the three terms in $V^{L B}$. The first term in curly braces would be the variance of the estimate if the trimming threshold $y_{1-p_{0}}$ were known. ${ }^{26}$ The second term in curly braces reflects the fact that the threshold is a quantile that needs to be estimated. Taken together, the first two terms are exactly equivalent to the expression given in Stigler [1973], who derives the asymptotic distribution of a one-sided " $p_{0}$-trimmed" mean, when $p_{0}$ is known. But $p_{0}$ is not known, and must be estimated, which is reflected in the third term. The Appendix contains the proof, which involves applying Theorem 7.2 of Newey and McFadden [1994], an asymptotic normality result for GMM estimators when the moment function is not smooth.

Estimation of the variances is easily carried out by replacing all of the above quantities (e.g., $E[S D]$, $y_{p_{0}}$ ) with either their sample analogs (e.g., $\left.\frac{1}{n} \sum S D, \widehat{y_{\widehat{p}}}\right)$. After assuming a finite second moment for $Y$, consistency follows because the resulting estimator is a continuous function of consistent estimators for each part.

There are two simple ways to compute confidence intervals. First, one can compute the interval $\widehat{\left[\Delta^{L B}\right.}$

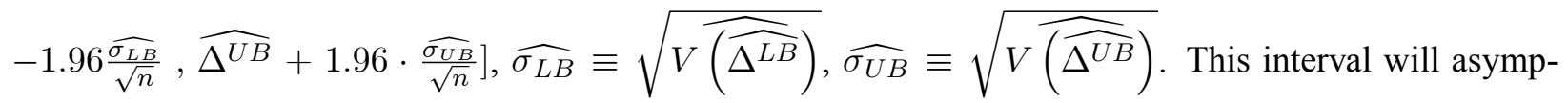
totically contain the region $\left[\Delta_{0}^{L B}, \Delta_{0}^{U B}\right]$ with at least 0.95 probability. ${ }^{27}$ Imbens and Manski [2004] point out that this same interval will contain the parameter $E\left[Y_{1}^{*}-Y_{1}^{*} \mid S_{0}=1, S_{1}=1\right]$ with an even greater probability, suggesting the confidence interval for the parameter will be narrower for the same coverage rate. The results of Imbens and Manski [2004] imply that a (smaller) interval of $\left[\widehat{\Delta^{L B}}-\bar{C}_{n} \cdot \frac{\widehat{\sigma_{L B}}}{\sqrt{n}}, \widehat{\Delta^{U B}}+\right.$ $\left.\bar{C}_{n} \frac{\widehat{\sigma_{U B}}}{\sqrt{n}}\right]$, where $\bar{C}_{n}$ satisfies

$$
\Phi\left(\bar{C}_{n}+\sqrt{n} \frac{\widehat{\Delta^{U B}}-\widehat{\Delta^{L B}}}{\max \left(\widehat{\sigma_{L B}}, \widehat{\sigma_{U B}}\right)}\right)-\Phi\left(-\bar{C}_{n}\right)=0.95
$$

\footnotetext{
${ }^{25}$ It is divided by $E[S(1-D)]$, because $n$ here is the total number of observations (selected and non-selected, treated and control).

26 The term $\frac{1}{E[S D]\left(1-p_{0}\right)}$ exists because $n$ is the size of the entire sample (both treatment and control, and all observations including those with missing outcomes).

27 To see this, note that $\operatorname{Pr}\left[\widehat{\Delta^{L B}}-1.96 \sigma^{L B}<\Delta_{0}^{L B}, \widehat{\Delta^{U B}}+1.96 \sigma^{U B}>\Delta_{0}^{U B}\right]$ is equivalent to $\operatorname{Pr}\left[\frac{\widehat{\Delta^{L B}}-\Delta_{0}^{L B}}{\sigma^{L B}}<1.96\right.$, $\left.\frac{\widehat{\Delta^{U B}}-\Delta_{0}^{U B}}{\sigma^{U B}}>-1.96\right]=1-\operatorname{Pr}\left[\frac{\widehat{\Delta^{L B}}-\Delta_{0}^{L B}}{\sigma^{L B}}>1.96\right]-\operatorname{Pr}\left[\frac{\widehat{\Delta^{U B}}-\Delta_{0}^{U B}}{\sigma^{U B}}<-1.96\right]+\operatorname{Pr}\left[\frac{\widehat{\Delta^{L B}}-\Delta_{0}^{L B}}{\sigma^{L B}}>1.96, \frac{\widehat{\Delta^{U B}}-\Delta_{0}^{U B}}{\sigma^{U B}}<-1.96\right]$, which is equal to $1-0.025-0.025+\operatorname{Pr}\left[\frac{\widehat{\Delta^{L B}}-\Delta_{0}^{L B}}{\sigma^{L B}}>1.96, \frac{\widehat{\Delta^{U B}}-\Delta_{0}^{U B}}{\sigma^{U B}}<-1.96\right]$, when $\frac{\widehat{\Delta^{L B}}-\Delta_{0}^{L B}}{\sigma^{L B}}, \frac{\widehat{\Delta^{U B}}-\Delta_{0}^{U B}}{\sigma^{U B}}$ is standard bivariate normal.
} 
can be computed, and it will contain the parameter $E\left[Y_{1}^{*}-Y_{1}^{*} \mid S_{0}=1, S_{1}=1\right]$ with a probability of at least 0.95 .

The interval of Imbens and Manski [2004] is more appropriate here since the object of interest is the treatment effect, and not the region of all rationalizable treatment effects. Nevertheless, for completeness, both intervals are reported in the presentation of the results.

\section{Empirical Results}

This section uses the trimming estimator to compute bounds on the treatment effect of the Job Corps on wage rates. The procedure is first employed for wages at week 208, 4-years after the date of random assignment. The width of the bounds are reasonably narrow and are suggestive of positive wage effects of the program. The bounds for the effect at week 208 do contain zero, but the bounds at week 90 do not. Overall, the evidence presented below points towards positive treatment effect, but not too much more than a 10 percent effect.

\section{V.A. Main Results at Week 208}

Table IV reports the estimates of the bounds of the treatment effect on wages at week 208. The construction of the bounds and their standard errors are illustrated in the table. Rows (iii) and (vi) report the means of log-wages for the treated and control groups. Rows (ii) and (v) report that about 61 percent of the treated group has non-missing wages while about 57 percent of the control group have non-missing wages. This implies a trimming proportion of about 6.8 percent of the treated group sample. The $p$ th quantile is about 1.64, and therefore the upper bound for the treated group is the mean after trimming the tail of the distribution below 1.64. ${ }^{28}$ After trimming, the resulting mean is about 2.09, and so the upper bound of the treatment effect $\widehat{\Delta^{U B}}$ is 0.093 (row (xi)). A symmetric procedure yields $\widehat{\Delta^{L B}}$ of -0.019 (row (xii)).

The width of these bounds is about 0.11 . Note that this is $1 / 14$ th the width of the bounds yielded by

\footnotetext{
28 The procedure can be easily adapted to the case of a dependent variable with discrete support. Suppose there are $n_{T}$ observations with non-missing wages in the treatment group. Then the data can be sorted by the dependent variable and the first $\left[p \cdot n_{T}\right]$ observations can be thrown out (where [.] is the greatest integer function), before calculating the trimmed mean. This procedure was used here, with the slight modification that the design weights were used, so the observations were dropped until the cumulated sum of the weights equaled the trimming proportion times the total sum of the weights in the treatment group.
} 
existing "imputation" procedures as reported in Table III (calculate 1.55 from rows (xi) and (xii)). The much larger interval in Table III is clearly driven by the relatively wide support of the outcome variable. ${ }^{29}$ The difference between the two sets of bounds make an important difference in gauging the magnitude of the effects of the program. From Table III, the negative region covered by the bounds is almost as large as the positive region contained by the bounds. In this sense, the bounds from Table III are almost as consistent with large negative effects as they are with large positive effects.

The width of the trimming bounds in Table IV are also narrow enough to rule out plausible effect sizes. For example, suppose the training component of the Job Corps program were ineffective at raising the marketable skills of the participants. We would then expect Job Corps to have a negative impact on wages, insofar as the time spent in the program caused a delay in accumulating labor market experience.

Suppose annual wage growth is about 8 percent a year, and the program group spent more time in education and training programs than the control group by an amount equivalent to 0.72 of a school year. ${ }^{30}$ If a full school year in training causes a year delay in earnings growth, this would imply Job Corps impact of about -0.058 . The lower bound in Table IV is -0.019 . Thus, the scenario described above is ruled out by the trimming bounds computed in Table IV. By contrast, an impact of -0.058 is easily contained by the support-dependent interval [-0.746,0.802] of Table III.

An impact of -0.058 is also outside the interval after accounting for sampling errors of the estimated bounds. The right side of Table IV illustrates the construction of these standard errors. For the estimate of the upper bound for the treatment group, Component 1 is the standard error associated with the first term in Equation (7). ${ }^{31}$ Component 2 reflects sampling error in estimating the trimming threshold. ${ }^{32}$ Component 3 reflects sampling error in estimating the trimming proportion. ${ }^{33}$ In this case, the largest source of the

\footnotetext{
${ }^{29}$ For a detailed theoretical discussion of how the imputation bounds (e.g. Table III) compare to the trimming bounds (e.g. Table IV) when the outcome is binary, see Lee [2002].

30 From Figure II, there appears to be about 40 percent nominal wage growth over 4 years. Inflation over that length of time in the late 1990s was about 9 percent (CPI-U for 1995: 152.4; for 1999; 166.6). Schochet et al. [2001] find that the Job Corps impact on time spent in any education and training programs amounted to about one school year per participant. The estimated impact per eligible applicant was 28 percent lower.

31 Specifically, it is the square root of the sample analog of $\frac{1}{n \cdot E[S D]\left(1-p_{0}\right)} \operatorname{Var}\left[Y \mid D=1, S=1, Y \geq y_{p_{0}}\right]$. In this case $\frac{1}{n \cdot E[S D]\left(1-p_{0}\right)}=1 / 3148$.

32 It is the square root of the sample analog of $\frac{1}{n E[S D]\left(1-p_{0}\right)}\left(y_{p_{0}}-\mu^{U B}\right)^{2} p_{0}$.

33 It is the square root of the sample analog of $\frac{1}{n}\left(y_{p_{0}}-\mu^{U B}\right)^{2}\left(\frac{(1-E[S \mid D=0])-p_{0}(1-E[D])}{E[D] \cdot E[S \mid D=0] \cdot(1-E[D])}\right)$.
} 
variance in the upper bound comes from the estimation of the trimming proportion. The total of 0.0092 is the square root of the sum of the squared components.

Doing a similar calculation for the lower bound, and then using the standard error on the mean for the

control group yields standard errors for $\widehat{\Delta^{U B}}$ and $\widehat{\Delta^{L B}}$ of 0.0123 and 0.0165 , as shown in the bottom of Table IV. These standard errors can then be used to compute two types of 95 percent confidence intervals. The first, covers the entire set of possible treatment effects with at least 0.95 probability, while the second interval, using the result from Imbens and Manski [2004], covers the true treatment effect at least 95 percent of the time. A plausible negative impact of -0.058 is outside both of these intervals.

\section{V.B. Using Covariates}

The width of the bounds can, in principle, be made narrower with the use of covariates. To gain intuition for the result, suppose half of the workers in the treatment group earn the wage $w^{H}$, while the other half earns the lower wage of $w^{L}$. The trimming procedure described in the previous sections suggest removing only low wage individuals, by a proportion $p_{0}$ to obtain an upper bound of the mean for the "inframarginally" selected. The trimmed mean will necessarily be larger.

Suppose now there is a baseline covariate $X$ that perfectly predicts whether an individual will earn $w^{H}$ or $w^{L}$. Then, due to the random assignment of treatment, Assumptions 1 and 2 also hold conditional on $X$. Therefore, the results in the previous section can be applied separately for the two types of workers. If, for both groups, the same proportion of observations are trimmed, the overall mean will not be altered by this trimming procedure..$^{34}$

More formally, consider the following alternative to Assumption 1,

Assumption 3 (Independence): Let $X$ be a vector of covariates, and let $\left(Y_{1}^{*}, Y_{0}^{*}, S_{1}, S_{0}, X\right)$ be independent of $D$.

In the case of the Job Corps Experiment, this assumption is valid when $X$ represents baseline characteristics; this is due to random assignment of treatment.

\footnotetext{
34 Strictly speaking, there are no upper or lower "tails", in this simple example, where the outcome is discrete. Nevertheless, the procedure can be adapted to discrete outcomes, as described in the subsection V.A.
} 
Proposition 4: Let $Y_{0}^{*}$ and $Y_{1}^{*}$ be continuous random variables. If Assumptions 3 and 2 hold, and without loss of generality $S_{1} \geq S_{0}$ with probability 1 , then $\Delta_{0}^{\overline{L B}}$ and $\Delta_{0}^{\overline{U B}}$ are sharp lower and upper bounds for the average treatment effect $E\left[Y_{1}^{*}-Y_{0}^{*} \mid S_{0}=1, S_{1}=1\right]$, where

$$
\begin{aligned}
\Delta_{0}^{\overline{L B}} & \equiv \int \Delta_{x}^{L B} d H(x) \\
\Delta_{0}^{\overline{U B}} & \equiv \int \Delta_{x}^{U B} d H(x), \text { where } H \text { is the cdf of } X \text { conditional on } D=0, S=1 \\
\Delta_{x}^{L B} & \equiv E\left[Y \mid D=1, S=1, Y \leq y_{1-p_{x}}, X=x\right]-E[Y \mid D=0, S=1, X=x] \\
\Delta_{x}^{U B} & \equiv E\left[Y \mid D=1, S=1, Y \geq y_{p_{x}}, X=x\right]-E[Y \mid D=0, S=1, X=x] \\
y_{q} & \equiv G_{x}^{-1}(q), \text { with } G_{x} \text { the cdf of } Y, \text { conditional on } D=1, S=1, X=x \\
p_{x} & \equiv \frac{\operatorname{Pr}[S=1 \mid D=1, X=x]-\operatorname{Pr}[S=1 \mid D=0, X=x]}{\operatorname{Pr}[S=1 \mid D=1, X=x]}
\end{aligned}
$$

The bounds are sharp in the sense that $\Delta_{0}^{\overline{L B}}\left(\Delta_{0}^{\overline{U B}}\right)$ is the largest (smallest) lower (upper) bound that is consistent with the observed data. Furthermore, $\Delta_{0}^{\overline{L B}} \geq \Delta_{0}^{L B}$ and $\Delta_{0}^{\overline{U B}} \leq \Delta_{0}^{U B}$.

The first part of the proposition follows from applying Proposition 1 conditionally on $X=x$. The second claim, that the width of the bounds must be narrower after utilizing the covariates, is seen by noting that any treatment effect that is consistent with an observed population distribution of $(Y, S, D, X)$, must also be consistent with the data after throwing away information on $X$, and observing only the distribution of $(Y, S, D)$. This necessity is strictly inconsistent with $\Delta_{0}^{\overline{U B}}>\Delta_{0}^{U B}$.

This modified procedure is implemented here as follows. First, the sample is split into 5 groups, based entirely on baseline characteristics $X$. Each of the five groups represent a different predicted wage, based on $X .{ }^{35}$ Then a trimming analysis is conducted for each of the five groups separately. Note that for each of the 5 groups, there is a different trimming proportion. The lower and upper bounds of the treatment group means, by each of the 5 groups, are given in the left and right columns of Table V, respectively. The lower bounds range from 1.81 to 2.11 , while the upper bounds range from 1.99 to 2.20 . The standard errors are computed for each group separately in the same manner as in Table IV.

To compute the bounds for the overall average $E\left[Y_{1}^{*} \mid S_{0}=1, S_{1}=1\right]$, the group-specific bounds must be averaged, weighted by the proportions $\operatorname{Pr}\left[\right.$ Group $\left.J \mid S_{0}=1, S_{1}=1\right]$. This is provided in the row la-

\footnotetext{
35 Week 208 wages were regressed on all baseline characteristics in Table I. The coefficients were then applied to all individuals to impute a predicted wage. The predicted wages were sorted, and the five groups were constructed according to the 20 th, 40 th, ... , 80th percentiles of the predicted wage distribution. Design weights were used for both the regression, and the categorization.
} 
belled "Total" ${ }^{36}$ This leads to an interval of $[-0.0103,0.0871]$. This interval is about 13 percent narrower than that reported in Table IV. The estimated variance for these overall averages is the sum of 1) the weighted-average of the group-specific variances and 2) the (weighted-) mean squared deviation of the group-specific estimates from the overall mean. This second term takes into account the sampling variability of the weights, as described in Chamberlain [1994]. ${ }^{37}$ These sampling errors lead to a 95 percent Imbens-Manski interval of $[-0.034,0.111]$.

By statistically ruling out any effect more negative than -0.034 , this suggests that after 4 years, the Job Corps enabled program group members to offset at least 40 percent and perhaps more of the potential 0.058 loss in wages due to lost labor market experience that could have been caused by the program.

\section{V.C. Effects by Time Horizon and Testable Implications}

An analysis of the bounds at different time horizons provides further evidence that the Job Corps program had a positive impact on wage rates. The analysis of Table IV was performed for impacts on wage rates at weeks 45, 90, 135, and 180, and these results are reported in Table VI. At each of the four time periods, the intervals defined by the bounds are more consistent with positive than negative impacts.

As would be expected, the width of the intervals are directly related to the treatment-control difference in the proportion missing. When the proportion is the largest, as at week 45, the range is [-0.074,0.127]. At week 180 , when the proportion is 0.0724 , the interval is [-0.033,0.087].

At week 90 , the trimming proportion is practically zero, and so the interval is $[0.042,0.043]$. The standard errors are larger for these bounds, even though they are quite similar to the untrimmed treatmentcontrol difference. This is partly due to the sampling error in the trimming proportion. Using these standard errors, a 95 percent confidence interval on the treatment effect would barely rule out a 0 effect at 90 weeks. On the other hand, if the trimming proportion is truly zero - and such a scenario cannot be statistically

\footnotetext{
36 There are slight differences in the number of observations in each group after trimming, for the upper and lower bounds. This is due to the use of the design weights.

37 The weighted mean of the 5 group-specific means, can be seen as a minimum distance estimator where the weights are the estimated proportions in each group.Chamberlain [1994] gives the asymptotic variance for this estimator even when the moment vector is mis-specified, as would be the case if the group-specific means are different. The asymptotic variance is the sum of two components: 1) the (observation-weighted) average of the asympotic variance for each group $\left(\Lambda_{1}\right.$ in Chamberlain [1994]), 2) the (observation-weighted) average squared deviation of each group's estimate from the "Total" mean $\left(\Lambda_{2}\right.$ in Chamberlain [1994]).
} 
ruled out - then a more efficient estimate of the treatment effect is given by the untrimmed estimate of 0.043 , which has a standard error of 0.011 .

Examining week 90 is helpful in providing some evidence on the plausibility of the monotonicity condition (Assumption 2). If at week 90, $E[S \mid D=1]-E[S \mid D=0]$ is truly zero, this implies that the average causal effect on sample selection $E\left[S_{1}-S_{0}\right]$ is zero. If monotonicity holds, then this can only be true if $S_{1}=S_{0}$ with probability $1 .^{38}$

If the only data that are observed is the triple $(Y, S, D)$, then it is impossible to test this monotonicity assumption. On the other hand, if there exist baseline characteristics $X$, as in the case of the Job Corps Experiment, then it is possible to test whether $S_{0}=S_{1}$ with probability 1 . That is, it is possible to test whether for each value of $X$, whether $\operatorname{Pr}[S=1 \mid D=1, X=x]=\operatorname{Pr}\left[S_{1}=1 \mid X=x\right]$ is equal to $\operatorname{Pr}[S=1 \mid D=0, X=x]=\operatorname{Pr}\left[S_{0}=1 \mid X=x\right]$, which should be the case for all $x$ if $S_{0}=S_{1}$ with probability 1 . Intuitively, if it was found that for some values of $X$, the treatment caused wages to be observed, while for other values of $X$, the treatment was found to cause wages to be missing, then Assumption 2 must not hold.

By Bayes' Rule and independence (Assumption 1), $\operatorname{Pr}[S=1 \mid D=1, X=x]=\operatorname{Pr}[S=1 \mid D=$ $0, X=x]$ for all $x$ implies that the distribution of $X$ conditional on $S=1, D=1$ should be the same as the distribution conditional on $S=1, D=0 .{ }^{39}$

A simple way to check this empirically is to examine the means of the variables in Table I, but conditional on having non-missing wages. This is done for week 90, and is reported in Appendix Table I. The differences between the treatment and control means for each variable are small and consistently statistically insignificant. A joint test of significance is given by a logistic regression of the treatment indicator on the baseline characteristics $X$, using a sample of all those with non-missing wages at week $90 .{ }^{40}$ The resulting test of all coefficients equaling zero yields a p-value of 0.851 . Thus, the data are consistent with

\footnotetext{
38 If $S_{1}=S_{0}$ with less than probability 1 , then there would be a nonzero probability of $S_{1}<S_{0}$, and it would be equal to the probability of $S_{0}>S_{1}$ - thus contradicting monotonicity - in order for $E\left[S_{1}-S_{0}\right]=0$.

39 This is because the density of $X$, conditional on $D$, does not depend on the value of $D$, and the probability of $S=1$ conditional on $D$ also does not depend on $D$, by assumption.

40 This is a valid test since in this context, $\operatorname{Pr}[S=1 \mid D=1, X=x]=\operatorname{Pr}[S=1 \mid D=0, X=x]$ for all $x$, is equivalent to the test $\operatorname{Pr}[D=1 \mid S=1, X=x] / \operatorname{Pr}[D=0 \mid S=1, X=x]=\operatorname{Pr}[D=0] / \operatorname{Pr}[D=1]$.
} 
the monotonicity condition holding at week 90 .

\section{Conclusion: Implications and Applications}

This paper focuses on an important issue in evaluating the impact of a job training program on wage rates - the sample selection problem. It is a serious issue even when the treatment of a training program is believed to be independent of all other factors, as was the case in the randomized experimental evaluation

of the U.S. Job Corps. Existing sample selection correction methods are infeasible due to the absence of plausible exclusion restrictions, and in this case, one cannot rely upon the boundedness of the outcome variable's support to yield informative bounds on the treatment effect of interest.

In order to estimate the impact of the Job Corps on wages, this paper develops a new method for bounding treatment effects in the presence of sample selection in the outcome. An appealing feature of the method is that the assumptions for identification, independence and monotonicity, are typically already assumed in standard models of the sample selection process, such as in Equation (1). In the case of randomized experiments, the independence assumption is satisfied, and as illustrated in the previous section, the existence of baseline characteristics suggest a limited test of monotonicity. More importantly, the bounding approach does not require any exclusion restrictions for the outcome equation. Nor do the trimming-bounds rely on the bounds of the support of the outcome variable.

The analysis using the proposed "trimming" bounds point to two substantive conclusions about the Job Corps. First, the evidence casts doubt on the notion that the program only raised earnings through raising labor force participation. Effects more negative than -0.034 can be statistically ruled out. If there were literally no wage effect, one might expect to see a more negative impact (perhaps around a -0.058 effect) due to lost labor market experience, since the youth applicants are on the steep part of their wage profile. More convincingly, at week 90 , the estimated lower bound is 0.042 , and this lower bound is on the margin of statistical significance at the 0.05 level.

Another reason to interpret the evidence as pointing to positive wage effects is that the lower bound is based on an extreme, and unintuitive assumption - that wage outcomes are perfectly negatively correlated 
with the propensity to be employed. From a purely theoretical standpoint, a simple labor supply model suggests that, all other things equal, those on the margin of being employed will have lowest wages, not the highest wages (i.e. the "reservation wage" will be the smallest wage that draws the individual into the labor force). In addition, the empirical evidence in Table II suggests that there is positive selection into employment: those who are predicted to have higher wages are more likely to be employed (i.e. $U$ and $V$ are positively correlated). If this is true, it seems relatively more plausible to trim the lower rather than the upper tail of the distribution to get an estimate of the treatment effect.

Second, the intervals provided here are comparable to rates of return found in the returns to education literature. At week 208, the point estimates an interval of [-0.0103,0.0871]. Program participants may be lagging behind their control counterparts by as much as 8 months in labor market experience due to enrollment in the program. As argued above, this could translate to as much as a 5.8 percent wage disadvantage even 4 years after random assignment, because many of the individuals in this sample are still on the steep part of their age-earnings profiles. Projecting to ages when the wage profile flattens leads to an interval of [.047,0.145]. A similar adjustment for week 90 wages yields an interval tightly centered around 0.10 . As found in a survey of studies that exploit institutional features of school systems [Card 1999], point estimates of the return to a single year of schooling range from 0.060 to $0.153 .{ }^{41}$ Thus, the magnitudes found in this analysis of the Job Corps are roughly consistent with viewing the program as a human capital investment of one year of schooling.

It should be emphasized that the trimming-bounds introduced here are specific neither to selection into employment nor to randomized experiments. For example, outcomes can be missing due to survey nonresponse (e.g., students not taking tests), sample attrition (e.g., inability to follow individuals over time), or other structural reasons (e.g., mortality). As long as the researcher believes that the sample selection process can be written as a model like Equation (1) or (5), the same trimming method can be applied. Also, the basis for matching estimators for evaluations is the weaker assumption that $\left(Y_{1}^{*}, Y_{0}^{*}\right)$ is independent of $D$, conditional on $X$, rather than Assumption 3. It is immediately clear that the trimming bounds proposed

\footnotetext{
41 See Table 4 in Card [1999].
} 
here can be applied even when $\left(Y_{1}^{*}, Y_{0}^{*}, S_{0}, S_{1}\right)$ is independent of $D$, but only conditional on $X$, as long as Assumption 2 holds conditionally on $X$. In this situation, the procedure described in sub-section V.B can be applied. ${ }^{42}$

\footnotetext{
42 But it should be noted that since the baseline characteristics $X$ would no longer be independent of the treatment, one could no longer use Remark 4 to test the monotonicity assumption.
} 


\section{Mathematical Appendix}

Lemma. Let $Y$ be a continuous random variable and a mixture of two random variables, with cdfs $M^{*}(y)$ and $N^{*}(y)$, and a known mixing proportion $p^{*} \in[0,1)$, so that we have $F^{*}(y)=p^{*} M^{*}(y)$ $+\left(1-p^{*}\right) N^{*}(y)$. Consider $G^{*}(y)=\max \left[0, \frac{F^{*}(y)-p^{*}}{1-p^{*}}\right]$, which is the cdf of $Y$ after truncating the $p^{*}$ lower tail of $Y$. Then $\int_{-\infty}^{\infty} y d G^{*}(y) \geq \int_{-\infty}^{\infty} y d N^{*}(y) \cdot \int_{-\infty}^{\infty} y d G^{*}(y)$ is a sharp upper bound for $\int_{-\infty}^{\infty} y d N^{*}(y)$.

Proof of Lemma. See Horowitz and Manski [1995], Corollary 4.1.

Proof of Proposition 1. It suffices to show that $\mu^{U B} \equiv E\left[Y \mid D=1, S=1, Y \geq y_{p_{0}}\right]$ is a sharp upper bound for $E\left[Y_{1}^{*} \mid S_{0}=1, S_{1}=1\right]$. A similar argument for the sharp lower bound would follow. Assumptions 1 and 2 imply that $p_{0}=\frac{\operatorname{Pr}[S=1 \mid D=1]-\operatorname{Pr}[S=1 \mid D=0]}{\operatorname{Pr}[S=1 \mid D=1]}=\frac{\operatorname{Pr}\left[S_{0}=0, S_{1}=1 \mid D=1\right]}{\operatorname{Pr}[S=1 \mid D=1]}$. Let $F(y)$ be the cdf of $Y$ conditional on $D=1, S=1$. Assumption 2 implies that $F(y)=p_{0} M(y)+\left(1-p_{0}\right) N(y)$, where $M(y)$ denotes the cdf of $Y_{1}^{*}$, conditional on $D=1, S_{0}=0, S_{1}=1$, and $N(y)$ denotes the cdf of $Y_{1}^{*}$, conditional on $D=1, S_{0}=1, S_{1}=1$. By Assumption 1, $N(y)$ is also the cdf of $Y_{1}^{*}$, conditional on $S_{0}=1, S_{1}=1$. By the Lemma, $\mu^{U B} \equiv \frac{1}{1-p_{0}} \int_{y_{p_{0}}}^{\infty} y d F(y) \geq \int_{-\infty}^{\infty} y d N(y)=E\left[Y_{1}^{*} \mid S_{0}=1, S_{1}=1\right]$.

To show that $\mu^{U B}$ equals the maximum possible value for $E\left[Y_{1}^{*} \mid S_{0}=1, S_{1}=1\right]$ that is consistent with the distribution of the observed data on $(Y, S, D)$, it must be shown that 1) conditional on $p_{0}, \mu^{U B}$ is a sharp upper bound, and 2) $p_{0}$ is uniquely determined by the data. 1) follows from the Lemma. 2) is true because the data yield a unique probability function $\operatorname{Pr}[S=s, D=d], s, d=0,1$, which uniquely determines $p_{0}$. Q.E.D.

Proof of Proposition 2. It is sufficient to prove consistency for the trimmed mean for the treatment group, and only for the lower bound, since a symmetrical argument will follow for the upper bound. Denote $\mu_{0} \equiv E\left[Y \mid D=1, S=1, Y \leq y_{p_{0}}\right]$ as the true lower bound of interest. Consistency follows from application of Theorem 2.6 of Newey and McFadden [1994], which applies to GMM estimators. Define the moment function

$$
g(z, \theta) \equiv\left(\begin{array}{c}
(Y-D \mu) S D \cdot 1\left[Y \leq y_{p}\right] \\
\left(1\left[Y>y_{p}\right]-p\right) S D \\
\left(S-D \alpha \frac{1}{1-p}\right) D \\
(S-(1-D) \alpha)(1-D)
\end{array}\right)
$$


where $\theta^{\prime}=\left(\mu, y_{p}, p, \alpha\right)^{\prime}, \theta_{0}^{\prime}=\left(\mu_{0}, y_{p_{0}}, p_{0}, \alpha_{0}\right)^{\prime}, \alpha_{0} \equiv E[S=1 \mid D=0]$, and $z^{\prime}=(Y, S, D)^{\prime}$. The estimator of $\mu_{0}$, the lower bound of $E\left[Y_{1}^{*} \mid S_{1}=1, S_{1}=1\right]$, as provided in Equation (6) is a solution to $\min _{\theta}\left(\sum g(z, \theta)\right)^{\prime} \cdot\left(\sum g(z, \theta)\right)$. It follows then that (i) through (iv) of Theorem 2.6 holds. Q.E.D.

Proof of Proposition 3. As in the proof above, it is sufficient to focus only on the asymptotic properties of the estimator of $\mu_{0}$. This estimator will be independent of that for the (untrimmed) control group mean. The proof follows by showing that the conditions of Theorem 7.2 of Newey and McFadden [1994] are satisfied.

Define $g_{0}(\theta) \equiv E[g(z, \theta)]$, and $\widehat{g}_{n}(\theta) \equiv n^{-1} \sum g(z, \theta)$. (i) of Theorem 7.2 holds. (iii) holds by assumption. (iv) holds by the central limit theorem. Let $G$ be the derivative of $g_{0}(\theta)$ at $\theta=\theta_{0}$. An explicit expression for $G$, a square matrix, is given below and will be shown to be nonsingular; hence (ii) holds as well.

The stochastic equicontinuity condition in (v) can shown to hold using Theorem 1 of Andrews [1994]. Assumption C of this theorem holds, and Assumption A holds with envelope $\bar{M}=\left|Y-D \mu_{0}\right|+|D| \sup _{\mu}|| \mu_{0}-$ $\mu \|$ for the first element, and 1 for the remaining elements of $g(z, \theta) . E|Y|^{2+\delta}<\infty$ for some $\delta>0$ implies that $E|\bar{M}|^{2+\delta}<\infty$ for some $\delta>0$, and therefore Assumption B holds as well.

From Theorem 7.2 of Newey and McFadden [1994], the asymptotic variance is $V^{L B}=G^{-1} \Sigma\left(G^{\prime}\right)^{-1}$ where $\Sigma$ is the asymptotic variance of $\widehat{g}_{n}\left(\theta_{0}\right)$. After letting $\gamma^{\prime} \equiv\left(\mu, y_{p}\right)^{\prime}$ and $\delta \equiv(p, \alpha)^{\prime}$, it can be shown that $G$ can be written as the partitioned matrix $\left(\begin{array}{cc}G_{\gamma} & G_{\delta} \\ 0 & M_{\delta}\end{array}\right)$ and $\Sigma$ can be partitioned as $\left(\begin{array}{cc}\Sigma_{1} & 0 \\ 0 & \Sigma_{2}\end{array}\right)$. The upper, left $2 \times 2$ block of $V^{L B}$ can then be shown to be equal to $G_{\gamma}^{-1} \Sigma_{1}\left(G_{\gamma}^{-1}\right)^{\prime}+G_{\gamma}^{-1} G_{\delta} M_{\delta}^{-1} \Sigma_{2}$. $\left(M_{\delta}^{-1}\right)^{\prime} G_{\delta}^{\prime}\left(G_{\gamma}^{-1}\right)^{\prime}$. The first term contains the variance of the trimmed mean, if the trimming proportion $p_{0}$ is known. The second term captures the variance due to the estimation of the trimming proportion.

Consider the first term. After computing $g_{0}(\theta), G_{\gamma}$ can be shown to equal

$$
E[S D]\left(\begin{array}{cc}
-\left(1-p_{0}\right) & \left(y_{p_{0}}-\mu_{0}\right) f\left(y_{p_{0}}\right) \\
0 & -f\left(y_{p_{0}}\right)
\end{array}\right),
$$

where $f(\cdot)$ is the density of $Y$ conditional on $D=1, S=1 . \Sigma_{1}$ is equal to

$$
\left(\begin{array}{cc}
\int_{-\infty}^{y_{1-p_{0}}}\left(y-\mu_{0}\right)^{2} f(y) d y \cdot E[S D] & 0 \\
0 & p_{0}\left(1-p_{0}\right) E[S D]
\end{array}\right)
$$


It follows that the upper left element of $G_{\gamma}^{-1} \Sigma_{1}\left(G_{\gamma}^{-1}\right)^{\prime}$ is

$$
\frac{1}{E[S D]\left(1-p_{0}\right)}\left\{\operatorname{Var}\left[Y \mid D=1, S=1, Y \leq y_{1-p_{0}}\right]+\left(y_{1-p_{0}}-\mu_{0}\right)^{2} p_{0}\right\}
$$

as stated in Equation (7).

Consider the second term. Direct calculation of $G_{\delta}, M_{\delta}$, and $\Sigma_{2}$ yield

$$
\begin{aligned}
& G_{\delta}=E[S D]\left(\begin{array}{cc}
0 & 0 \\
-1 & 0
\end{array}\right), M_{\delta}=\left(\begin{array}{cc}
-E[D] \alpha_{0} \frac{1}{\left(1-p_{0}\right)^{2}} & -E[D] \frac{1}{1-p_{0}} \\
0 & -(1-E[D])
\end{array}\right) \\
& \Sigma_{2}=\left(\begin{array}{cc}
\frac{\alpha_{0}}{1-p_{0}}\left(1-\frac{\alpha_{0}}{1-p_{0}}\right) E[D] & 0 \\
0 & \alpha_{0}\left(1-\alpha_{0}\right)(1-E[D])
\end{array}\right)
\end{aligned}
$$

After simplifying terms, it follows that the upper left element of $G_{\gamma}^{-1} G_{\delta} M_{\delta}^{-1} \Sigma_{2}\left(M_{\delta}^{-1}\right)^{\prime} G_{\delta}^{\prime}\left(G_{\gamma}^{-1}\right)^{\prime}$ is equal to

$$
\left(y_{p_{0}}-\mu_{0}\right)^{2}\left(\frac{\left(1-\alpha_{0}\right)-p_{0}(1-E[D])}{E[D] \alpha_{0}(1-E[D])}\right)
$$

as stated in Equation (7). Q.E.D. 


\section{References}

Ahn, Hyungtaik and James Powell, "Semiparametric Estimation of Censored Selection Models with a Nonparametric Selection Mechanism," Journal of Econometrics, LVIII (1993), 3-29.

Andrews, D. and M. Schafgans, "Semiparametric Estimation of the Intercept of a Sample Selection Model," Review of Economic Studies, LXV (1998), 497-517.

Andrews, Donald W. K., "Empirical Process Methods in Econometrics," in Robert F. Engle and Daniel L. McFadden, eds., Handbook of Eonometrics, Vol. 4 (Amsterdam: North Holland, 1994).

Angrist, Joshua, "Conditional Independence in Sample Selection Models," Economics Letters, LIV (1997), 103-112.

__ Guido Imbens, and D. Rubin, "Identification of Causal Effects Using Instrumental Variables," Journal of the American Statistical Association, XCI (1996), 444-455.

Balke, A. and J. Pearl, "Bounds on Treatment Effects from Studies With Imperfect Compliance," Journal of the American Statistical Association, XCII (1997), 1171-1177.

Barnow, B., "The impact of CETA on the post-program earnings of participants," Journal of Human Resources, XXII (1987), 157-193.

Bloom, H., "Accounting for No-Shows in Experimental Evaluation Designs," Evaluation Review, VIII (1984), 225-246.

Burghardt, John, Peter Z. Schochet, Sheena McConnell, Terry Johnson, R. Mark Gritz, Steven Glazerman, John Homrighausen, and Russell Jackson, "Does Job Corps Work? Summary of the National Job Corps Study," Report, Washington, DC, Mathematica Policy Research, Inc., 2001.

Card, David, "The Causal Effect of Education on Earnings," in Orley Ashenfelter and David Card, eds., Handbook of Labor Economics, Vol. 3A (Amsterdam: North Holland, 1999).

Chamberlain, Gary, "Quantile Regression, Censoring, and the Structure of Wages," in C. A. Sims, ed., Advances in Econometrics, Sixth World Congress, Vol. 1 (Cambridge: Cambridge University Press, 1994).

Das, Mitali, Whitney K. Newey, and Francis Vella, "Nonparametric Estimation of Sample Selection Models," Review of Economic Studies, LXX (2003), 33-58.

Heckman, James and R. Robb, "Alternative methods for solving the problem of selection bias in evaluating the impact of treatments on outcomes," in H. Wainer, ed., Drawing inferences from self-selected samples, (New York, NY: Springer, 1986).

Heckman, James J., "Shadow Prices, Market Wages, and Labor Supply,” Econometrica, XLII (1974), 679-694.

__ ,"Sample Selection Bias as a Specification Error," Econometrica, XLVII (1979), 153-161.

__ , "Varieties of Selection Bias," American Economic Review, LXXX (1990), 313-318.

and Edward Vytlacil, "Local Instrumental Variables and Semiparametric Estimation and Latent Variable Models for Identifying and Bounding Treatment Effects," Proceedings of the National Academy of Sciences, XCVI (1999), 4730-4734.

and ___ "Instrumental Variables, Selection Models, and Tight Bounds on the Average Treatment Effect,” Technical Working Paper 259, National Bureau of Economic Research, 2000.

and ___ , "Local Instrumental Variables," Technical Working Paper 252, National Bureau of Economic Research, 2000.

_ , Robert J. LaLonde, and James A. Smith, "The Economics and Econometrics of Active Labor Market Programs," in Orley Ashenfelter and David Card, eds., Handbook of Labor Economics, Vol. 3A (Amsterdam: North Holland, 1999).

Hollister, R., P. Kemper, and R. Maynard, The National Supported Work Demonstration (Madison, WI: 
University of Wisconsin Press, 1984).

Horowitz, Joel L. and Charles F. Manski, "Identification and Robustness with Contaminated and Corrupted Data," Econometrica, LXIII (1995), 281-302.

and __ "Nonparametric Analysis of Randomized Experiments with Missing Covariate and Outcome Data," Journal of the American Statistical Association, XCV (2000), 77-84.

and ___ "Rejoinder: Nonparametric Analysis of Randomized Experiments with Missing Covariate and Outcome Data," Journal of the American Statistical Association, XCV (2000), 87.

Imbens, Guido and Joshua Angrist, "Identification and Estimation of Local Average Treatment Effects," Econometrica, LXII (1994), 467-476.

Imbens, Guido W. and Charles F. Manski, "Confidence Intervals for Partially Identified Parameters," Econometrica, LXXII (2004), 1845-1857.

Kiefer, N., The economic benefits of four employment and training programs (New York, NY: Garland Publishing, 1979).

Krueger, Alan B. and Diane M. Whitmore, "The Effect of Attending a Small Class in the Early Grades on College-Test Taking and Middle School Test Results: Eviddence from Project STAR," Economic Journal, CXI (2001), 1-28.

Lee, David S., "Trimming for Bounds on Treatment Effects with Missing Outcomes," Center for Labor Economics Working Paper 38, Berkeley, University of California, 2002.

Martin, John P., "What works among Active Labour Market Policies: Evidence from OECD Countries' Experiences," OECD Economic Studies, XXX (2000), 79-113.

Newey, Whitney K. and Daniel McFadden, "Large Sample Estimation and Hypothesis Testing," in Robert F. Engle and Daniel L. McFadden, eds., Handbook of Eonometrics, Vol. 4 (Amsterdam: North Holland, 1994).

Robins, J., "The Analysis of Randomized and Non-Randomized AIDS Treatment Trials Using a New Approach to Causal Inference in Longitudinal Studies," in L. Sechrest, H. Freeman, and A. Mulley, eds., Health Service Research Methodology: A Focus on AIDS, (Washington, DC: U.S. Public Health Service, 1989).

Rubin, D., "Inference and Missing Data," Biometrika, LXIII (1976), 581-592.

Schochet, Peter Z., Jeanne Bellotti Ruo-Jiao Cao, Steven Glazerman, April Grady, Mark Gritz, Sheena McConnell, Terry Johnson, and John Burghardt, "National Job Corps Study: Data Documentation and Public Use Files, Volume I," Documentation, Washington, DC, Mathematica Policy Research, Inc., 2003.

_ John Burghardt, and Steven Glazerman, "National Job Corps Study: The Impacts of Job Corps on Participants' Employment and Related Outcomes," Report, Washington, DC, Mathematica Policy Research, Inc., 2001.

Stigler, Stephen M., "The Asymptotic Distribution of the Trimmed Mean," Annals of Statistics, I (1973), 472-477.

U.S. Department of Labor, "Summary of Budget Authority, Fiscal Years 2004-2005," Table, Employment and Training Administration, 2005.

__ , "What is Job Corps?," Web Page, Employment and Training Administration, 2005. <http://jobcorps. doleta.gov/about.cfm>.

Vytlacil, Edward, “Independence, Monotonicity, and Latent Index Models: An Equivalence Result," Econometrica, LXX (2002), 331-341. 
Figure I: Impact of Job Corps on Weekly Earnings

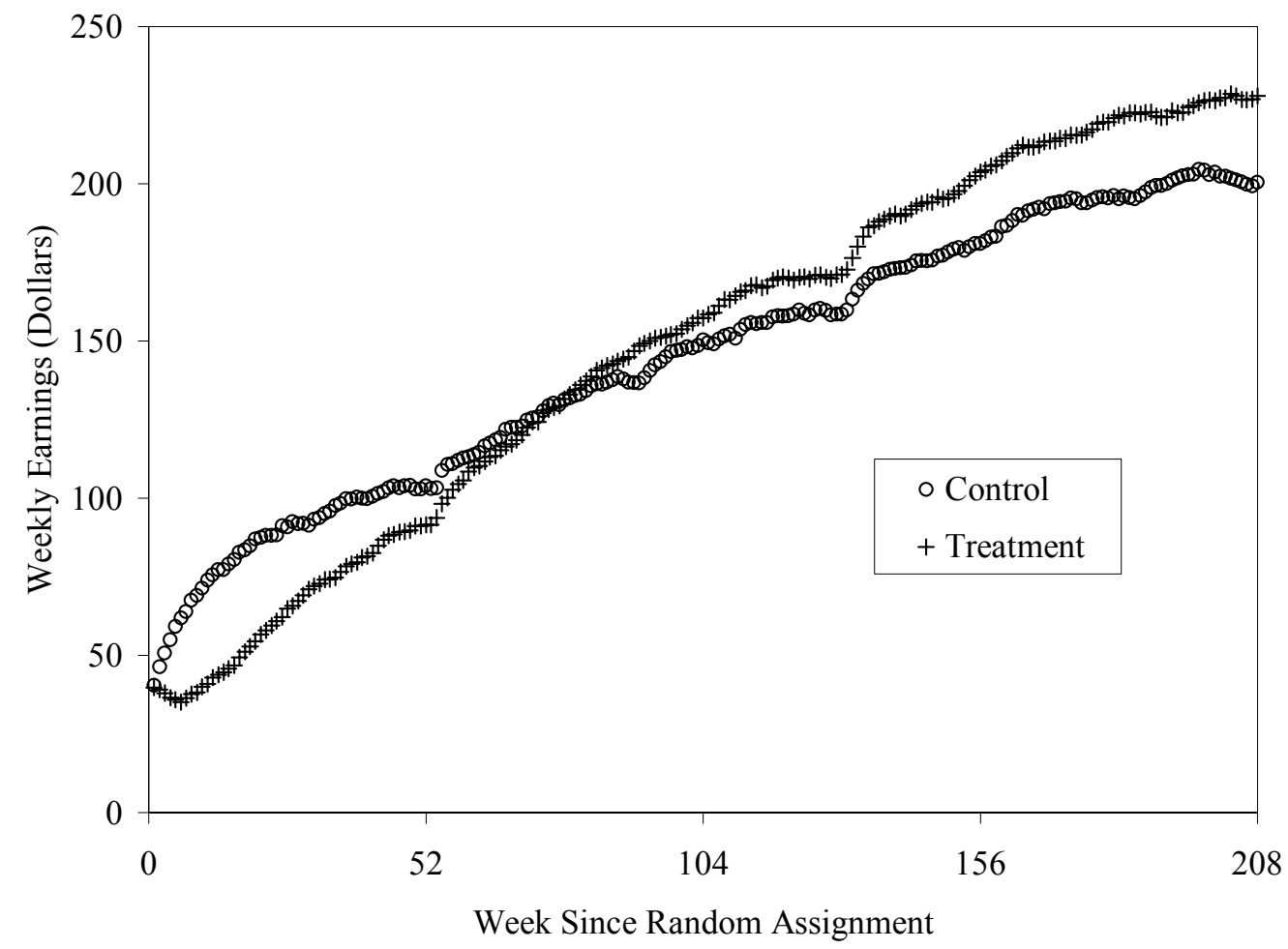

Figure II: Impact of Job Corps on Employment Rates

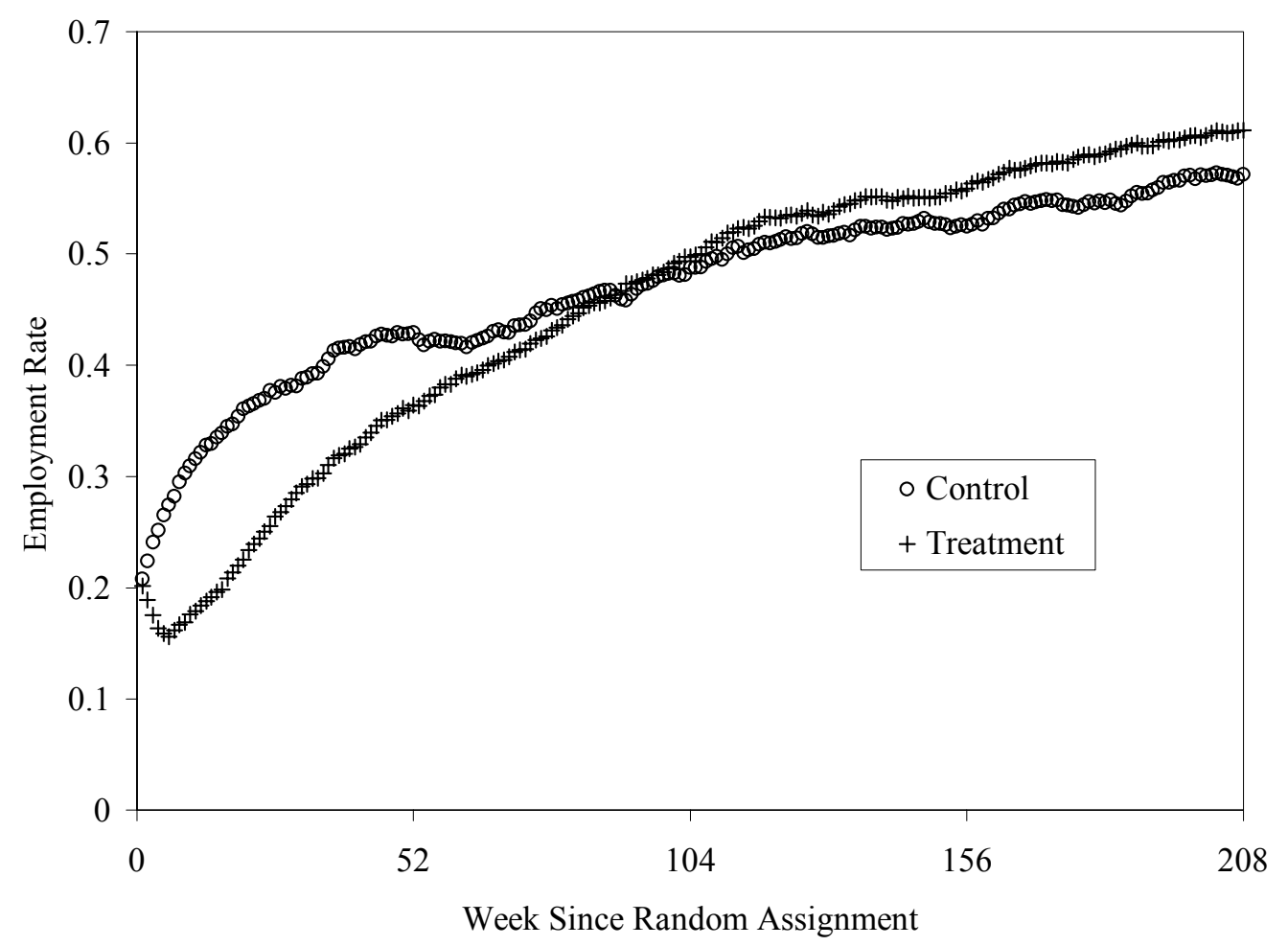


Figure III: Differences in Log(Hourly Wage), Conditional on Employment

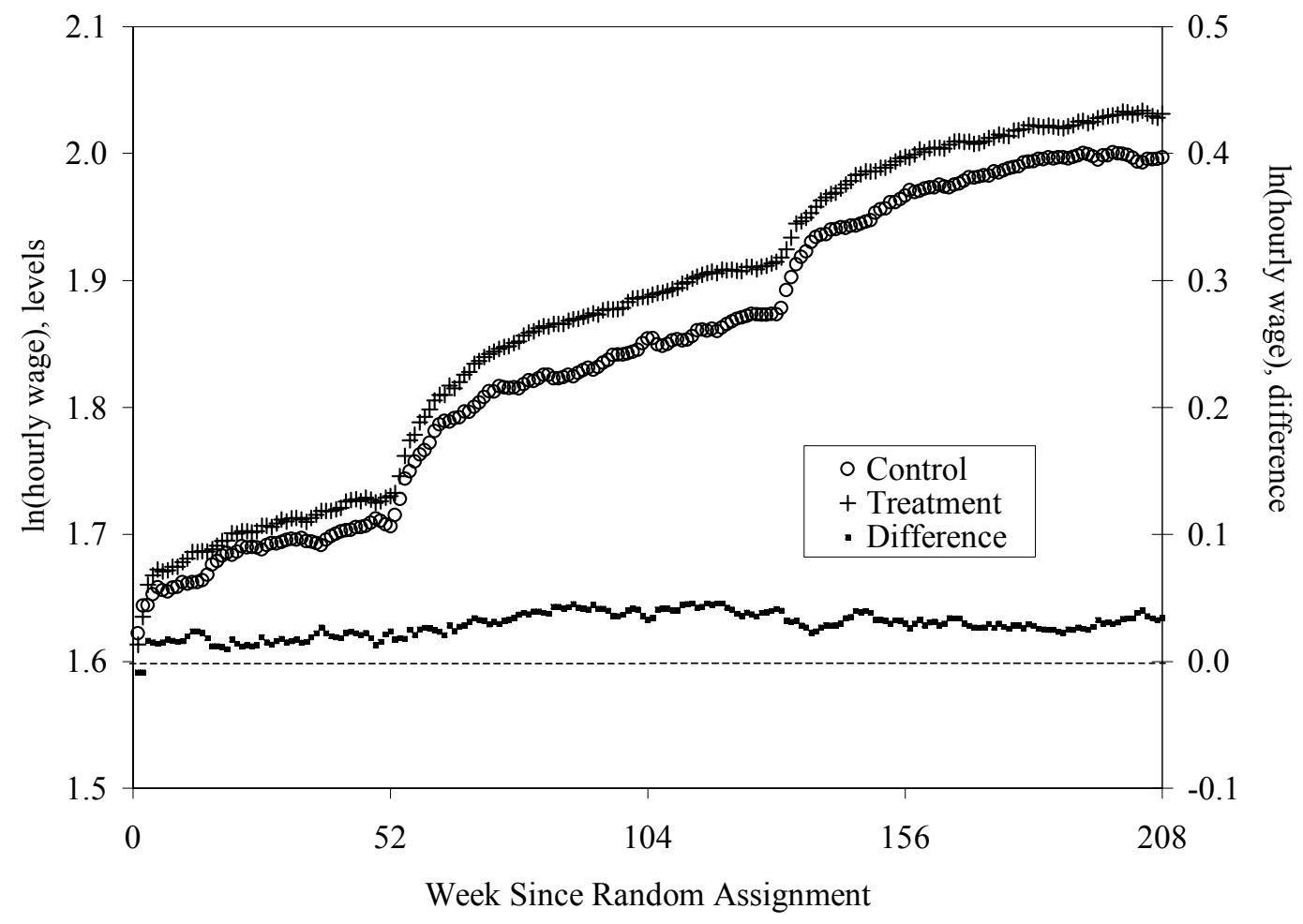




\begin{tabular}{|c|c|c|c|c|c|c|c|c|}
\hline \multirow[b]{2}{*}{ Variable } & \multicolumn{3}{|l|}{ Control } & \multicolumn{3}{|l|}{ Program } & \multicolumn{2}{|l|}{ Difference } \\
\hline & $\begin{array}{l}\text { Prop. Non- } \\
\text { Missing }\end{array}$ & Mean & Std. Dev. & $\begin{array}{l}\text { Prop. Non- } \\
\text { Missing }\end{array}$ & Mean & Std. Dev. & Diff. & Std. Err. \\
\hline Female & 1.00 & 0.458 & 0.498 & 1.00 & 0.452 & 0.498 & -0.006 & 0.011 \\
\hline Age at Baseline & 1.00 & 18.351 & 2.101 & 1.00 & 18.436 & 2.159 & 0.085 & 0.045 \\
\hline White, Non-Hispanic & 1.00 & 0.263 & 0.440 & 1.00 & 0.266 & 0.442 & 0.002 & 0.009 \\
\hline Black, Non-Hispanic & 1.00 & 0.491 & 0.500 & 1.00 & 0.493 & 0.500 & 0.003 & 0.011 \\
\hline Hispanic & 1.00 & 0.172 & 0.377 & 1.00 & 0.169 & 0.375 & -0.003 & 0.008 \\
\hline Other Race/Ethnicity & 1.00 & 0.074 & 0.262 & 1.00 & 0.072 & 0.258 & -0.002 & 0.006 \\
\hline Never married & 0.98 & 0.916 & 0.278 & 0.98 & 0.917 & 0.275 & 0.002 & 0.006 \\
\hline Married & 0.98 & 0.023 & 0.150 & 0.98 & 0.020 & 0.139 & -0.003 & 0.003 \\
\hline Living together & 0.98 & 0.040 & 0.197 & 0.98 & 0.039 & 0.193 & -0.002 & 0.004 \\
\hline Separated & 0.98 & 0.021 & 0.144 & 0.98 & 0.024 & 0.154 & 0.003 & 0.003 \\
\hline Has Child & 0.99 & 0.193 & 0.395 & 0.99 & 0.189 & 0.392 & -0.004 & 0.008 \\
\hline Number of children & 0.99 & 0.268 & 0.640 & 0.99 & 0.270 & 0.650 & 0.002 & 0.014 \\
\hline Education & 0.98 & 10.105 & 1.540 & 0.98 & 10.114 & 1.562 & 0.009 & 0.033 \\
\hline Mother's Educ. & 0.81 & 11.461 & 2.589 & 0.82 & 11.483 & 2.562 & 0.022 & 0.061 \\
\hline Father's Educ. & 0.61 & 11.540 & 2.789 & 0.62 & 11.394 & 2.853 & -0.146 & 0.077 \\
\hline Ever Arrested & 0.98 & 0.249 & 0.432 & 0.98 & 0.249 & 0.432 & -0.001 & 0.009 \\
\hline Household Inc: $<3000$ & 0.65 & 0.251 & 0.434 & 0.63 & 0.253 & 0.435 & 0.002 & 0.012 \\
\hline $3000-6000$ & 0.65 & 0.208 & 0.406 & 0.63 & 0.206 & 0.405 & -0.002 & 0.011 \\
\hline $6000-9000$ & 0.65 & 0.114 & 0.317 & 0.63 & 0.117 & 0.321 & 0.003 & 0.008 \\
\hline $9000-18000$ & 0.65 & 0.245 & 0.430 & 0.63 & 0.245 & 0.430 & 0.000 & 0.011 \\
\hline$>18000$ & 0.65 & 0.182 & 0.386 & 0.63 & 0.179 & 0.383 & -0.003 & 0.010 \\
\hline Personal Inc: $<3000$ & 0.92 & 0.789 & 0.408 & 0.92 & 0.789 & 0.408 & -0.001 & 0.009 \\
\hline $3000-6000$ & 0.92 & 0.131 & 0.337 & 0.92 & 0.127 & 0.334 & -0.003 & 0.007 \\
\hline $6000-9000$ & 0.92 & 0.046 & 0.209 & 0.92 & 0.053 & 0.223 & 0.007 & 0.005 \\
\hline$>9000$ & 0.92 & 0.034 & 0.181 & 0.92 & 0.031 & 0.174 & -0.003 & 0.004 \\
\hline \multicolumn{9}{|l|}{ At Baseline: } \\
\hline Have Job & 0.98 & 0.192 & 0.394 & 0.98 & 0.198 & 0.398 & 0.006 & 0.009 \\
\hline Mos. Empl. Prev. Yr. & 1.00 & 3.530 & 4.238 & 1.00 & 3.596 & 4.249 & 0.066 & 0.091 \\
\hline Had Job, Prev. Yr. & 0.98 & 0.627 & 0.484 & 0.98 & 0.635 & 0.482 & 0.007 & 0.010 \\
\hline Earnings, Prev. Yr. & 0.93 & 2810.482 & 4435.616 & 0.94 & 2906.453 & 6401.328 & 95.971 & 117.097 \\
\hline Usual Hours/Week & 1.00 & 20.908 & 20.704 & 1.00 & 21.816 & 21.046 & $0.908 *$ & 0.446 \\
\hline Usual Wkly Earnings & 1.00 & 102.894 & 116.465 & 1.00 & 110.993 & 350.613 & 8.099 & 5.093 \\
\hline \multicolumn{9}{|c|}{ After Random Assignment: } \\
\hline Week 52 Wkly Hours & 1.00 & 17.784 & 23.392 & 1.00 & 15.297 & 22.680 & $-2.487 *$ & 0.495 \\
\hline Week 104 Wkly Hours & 1.00 & 21.977 & 26.080 & 1.00 & 22.645 & 26.252 & 0.668 & 0.560 \\
\hline Week 156 Wkly Hours & 1.00 & 23.881 & 26.151 & 1.00 & 25.879 & 26.574 & $1.997 *$ & 0.563 \\
\hline Week 208 Wkly Hours & 1.00 & 25.833 & 26.250 & 1.00 & 27.786 & 25.745 & $1.953 *$ & 0.558 \\
\hline Week 52 Wkly. Earn. & 1.00 & 103.801 & 159.893 & 1.00 & 91.552 & 149.282 & $-12.249 *$ & 3.335 \\
\hline Week 104 Wkly Earn. & 1.00 & 150.407 & 210.241 & 1.00 & 157.423 & 200.266 & 7.015 & 4.417 \\
\hline Week 156 Wkly Earn. & 1.00 & 180.875 & 224.426 & 1.00 & 203.714 & 239.802 & $22.839 *$ & 4.936 \\
\hline Week 208 Wkly Earn. & 1.00 & 200.500 & 230.661 & 1.00 & 227.912 & 250.222 & $27.412 *$ & 5.106 \\
\hline Total Earn. (4 years) & 1.00 & 30007 & 26894 & 1.00 & 30800 & 26437 & 794 & 572 \\
\hline Number of Obs & 3599 & & & 5546 & & & & \\
\hline
\end{tabular}


Table II: Logit of Employment in Week 208 on Baseline Characteristics

\begin{tabular}{|c|c|c|c|}
\hline Variable & Estimate & Variable & Estimate \\
\hline \multirow[t]{2}{*}{ Treatment Status } & $0.172 *$ & Household Inc: & \\
\hline & $(0.046)$ & $3000-6000$ & 0.033 \\
\hline \multirow[t]{2}{*}{ Female } & $-0.253 *$ & & $(0.085)$ \\
\hline & $(0.051)$ & $6000-9000$ & $0.213 *$ \\
\hline \multirow[t]{2}{*}{ Age at Baseline } & 0.027 & & $(0.104)$ \\
\hline & $(0.014)$ & $9000-18000$ & 0.149 \\
\hline \multirow[t]{2}{*}{ Black, Non-Hispanic } & $-0.471 *$ & & $(0.086)$ \\
\hline & $(0.060)$ & $>18000$ & 0.103 \\
\hline \multirow[t]{2}{*}{ Hispanic } & $-0.225 *$ & & $(0.095)$ \\
\hline & $(0.077)$ & Personal Inc: & \\
\hline \multirow[t]{2}{*}{ Other Race/Ethnicity } & $-0.412 *$ & $3000-6000$ & 0.105 \\
\hline & $(0.099)$ & & $(0.080)$ \\
\hline \multirow[t]{2}{*}{ Married } & -0.193 & $6000-9000$ & 0.180 \\
\hline & $(0.175)$ & & $(0.127)$ \\
\hline \multirow[t]{2}{*}{ Living together } & 0.106 & $>9000$ & 0.197 \\
\hline & $(0.130)$ & & $(0.162)$ \\
\hline \multirow[t]{2}{*}{ Separated } & -0.261 & At Baseline: & \\
\hline & $(0.165)$ & Have Job & $0.218 *$ \\
\hline \multirow[t]{2}{*}{ Has Child } & 0.121 & & $(0.071)$ \\
\hline & $(0.114)$ & Mos. Empl. Prev. Yr. & $0.049 *$ \\
\hline \multirow[t]{2}{*}{ Number of children } & -0.031 & & $(0.011)$ \\
\hline & $(0.070)$ & Had Job, Prev. Yr. & $0.306 *$ \\
\hline \multirow[t]{2}{*}{ Education } & $0.104 *$ & & $(0.091)$ \\
\hline & $(0.019)$ & Earnings, Prev. Yr. $\left({ }^{*} 10000\right)$ & 0.012 \\
\hline \multirow[t]{2}{*}{ Mother's Educ. } & 0.007 & & $(0.120)$ \\
\hline & $(0.012)$ & Usual Hours/Week $(* 10000)$ & -26.580 \\
\hline \multirow[t]{2}{*}{ Father's Educ. } & -0.006 & & $(19.508)$ \\
\hline & $(0.012)$ & Usual Wkly Earnings $(* 10000)$ & 0.845 \\
\hline \multirow[t]{2}{*}{ Ever Arrested } & $-0.223 *$ & & $(1.990)$ \\
\hline & $(0.055)$ & Constant & $\begin{array}{l}-1.288 * \\
(0.285)\end{array}$ \\
\hline
\end{tabular}

Note: $\mathrm{N}=9415$. Robust standard errors in parentheses. Table reports are (log-odds) coefficients from a logit of employment (positive hours) in week 208 on treatment status and baseline characteristics. * denotes statistically significance at the 0.05 (or less). 
Table III: Bounds on Treatment Effects for Week $208 \ln$ (wage)

Utilizing Bounds of Support (Horowitz and Manski)

\begin{tabular}{|c|c|c|c|}
\hline (i) & Control Group & Observations & 3599 \\
\hline (ii) & & Employment Rate & 0.566 \\
\hline (iii) & & Mean log(wage) & 1.997 \\
\hline (iv) & & Upper Bound & 2.332 \\
\hline (v) & & Lower Bound & 1.520 \\
\hline (vi) & Treatment Group & Observations & 5546 \\
\hline (vii) & & Employment Rate & 0.607 \\
\hline (viii) & & Mean log(wage) & 2.031 \\
\hline (ix) & & Upper Bound & 2.321 \\
\hline (x) & & Lower Bound & 1.586 \\
\hline (xi) & Difference & Upper Bound: (ix) - (v) & 0.802 \\
\hline (xii) & & Lower Bound: (x) - (iv) & -0.746 \\
\hline
\end{tabular}

Note: .90 and 2.77 are the lower and upper bounds of the support of ln(hourly wage) in Week 208 after random assignment. (iv) $=($ ii) $*($ iii) $+[1-($ ii) $] * 2.77$. (v) $=($ ii) $*($ iii $)+$ [1-(ii) $]^{*}(.90)$. Rows (ix) and (x) are defined analogously. 
Table IV: Bounds on Treatment Effects for $\ln$ (wage) in Week 208 using Trimming Procedure

Control

(i) Number of Observations

3599

Control Standard Error

(ii) Proportion Non-missing

0.566

Std. Error

0.0082

(iii) Mean $\ln$ (wage) for employed

1.997

Treatment (iv) Number of Observations

5546

Treatment UB Standard Error

(v) Proportion Non-missing

0.607

(vi) Mean $\ln$ (wage) for employed

2.031

$\mathrm{p}=[(\mathrm{v})-(\mathrm{ii})] /(\mathrm{v})$

0.068

(vii) pth quantile

1.636

(viii) Trimmed Mean: $E\left[Y \mid Y>y_{p}\right]$

2.090

(ix) (1-p)th quantile

2.768

(x) Trimmed Mean: $\mathrm{E}\left[\mathrm{Y} \mid \mathrm{Y}<\mathrm{y}_{1-\mathrm{p}}\right]$

1.978

Component 1

0.0053

Component 2

0.0021

Component 3

0.0072

Total

0.0092

Treatment LB Standard Error

Component $1 \quad 0.0058$

Component $2 \quad 0.0037$

Component $3 \quad 0.0125$

Total

0.0143

Effect

Effect (xi) Upper Bound Estimate $=(8)-(3) \quad 0.093$

(xiii) UB Std.Err.

0.0123

(xii) Lower Bound Estimate $=(10)-(3) \quad-0.019$

(xiv) LB Std.Err.

0.0165

Confidence Interval $1=[($ xii $)-1.96 *(x i v),(x i)+1.96 *(x i i i)]$

Confidence Interval 2 (Imbens and Manski) $=\left[\left(\right.\right.$ xii) $\left.-1.645^{*}(x i v),(x i)+1.645^{*}(x i i i)\right]$

$[-0.052,0.117]$

$[-0.046,0.113]$

Note: After trimming, there are 3148 (3142) observations remaining in the treatment group after trimming the lower $\mathrm{p}$ (upper 1-p) of the distribution. These numbers are not indentical due to using the design weights. For the Upper Bound Standard Error, Component 1 is the usual standard error of the mean, using the trimmed sample. Component 2 is the square root of $\mathrm{p}^{*}(1 / 3148) *\left\{(\text { viii)-(vii) }\}^{2}\right.$. Component 3 is the square root of $\{(1-(\mathrm{v})) /(1-.491)$ $\mathrm{p}\}^{*}\{1 /((\mathrm{v}) * 5546)\}^{*}\left\{(\text { viii)-(vii) }\}^{2}\right.$ where 0.491 is the (weighted) proportion of the entire sample that is in the treatment group. "Total" refers to the square root of the sum the squared components. The entries for the Treatment LB Standard Error are defined analogously. (xiii) and (xiv) are the square root of the sum of the squared standard errors for the treatment UB (or LB) and control group. For the Imbens and Manski confidence interval 1.645 satisifies $\Phi(1.645+((x i)-(x i i)) /(\max ((x i i i),(x i v)))-\Phi(-1.645)=0.95$, where $\Phi$ is the standard normal cdf. See Imbens and Manski (2004) for details. 
Table V: Bounds on Treatment Effects for $\ln$ (wage) in Week 208

Trimming Procedure using Baseline Covariates

Lower Bound for Treatment Mean

Group Estimate Std. Error Obs.

$\begin{array}{llll}1 & 1.814 & 0.022 & 463\end{array}$

$2 \quad 1.960$

0.036

1.941

0.021

2.030

0.026

0.023

5

2.111

1.987

0.012

3137

Effect Lower Bound for Effect

$-0.0103$

Upper Bound for Treatment Mean

Estimate Std. Error Obs.

$\begin{array}{lll}1.994 & 0.020 & 468\end{array}$

$1.984 \quad 0.047 \quad 584$

$2.059 \quad 0.020 \quad 631$

$\begin{array}{lll}2.120 & 0.019 & 711\end{array}$

$\begin{array}{lll}2.204 & 0.020 & 758\end{array}$

$2.084 \quad 0.012$

3152

Upper Bound for Effect

$0.0871 \quad 0.0145$

Note: Trimming procedure from Table III applied separately to each Group (defined in text). "Total" estimates are means of the 5 groups using the observations as weights. Asymptotic variance for "Total" is computed according to Chamberlain (1993): it is the (observation-weighted) average of the asymptotic variance for each group plus the (observation-weighted) average squared deviation of each group's estimate from the "Total" mean. Control mean, (iii) in Table IV, is then subtracted to obtain bounds on the treatment effect. 
Table VI: Treatment Effect Estimates and Bounds, by Week

\begin{tabular}{|c|c|c|c|c|c|c|}
\hline & \multicolumn{2}{|c|}{ Fraction Non-missing } & \multirow[b]{2}{*}{$\begin{array}{l}\text { Trimming } \\
\text { Proportion }\end{array}$} & \multicolumn{3}{|l|}{ Effect } \\
\hline & Control & Treatment & & Untrimmed & $\begin{array}{l}\text { Lower } \\
\text { Bound }\end{array}$ & $\begin{array}{l}\text { Upper } \\
\text { Bound }\end{array}$ \\
\hline Week 45 & 0.4223 & 0.3424 & $\begin{array}{r}0.1892 \\
(0.0242)\end{array}$ & $\begin{array}{r}0.022 \\
(0.011)\end{array}$ & $\begin{array}{r}-0.074 \\
(0.014)\end{array}$ & $\begin{array}{r}0.127 \\
(0.015)\end{array}$ \\
\hline Week 90 & 0.4600 & 0.4601 & $\begin{array}{r}0.0003 \\
(0.0204)\end{array}$ & $\begin{array}{r}0.043 \\
(0.011)\end{array}$ & $\begin{array}{r}0.042 \\
(0.021)\end{array}$ & $\begin{array}{r}0.043 \\
(0.023)\end{array}$ \\
\hline Week 135 & 0.5173 & 0.5451 & $\begin{array}{r}0.0509 \\
(0.0168)\end{array}$ & $\begin{array}{r}0.028 \\
(0.011)\end{array}$ & $\begin{array}{r}-0.016 \\
(0.019)\end{array}$ & $\begin{array}{r}0.076 \\
(0.014)\end{array}$ \\
\hline Week 180 & 0.5403 & 0.5825 & $\begin{array}{r}0.0724 \\
(0.0154)\end{array}$ & $\begin{array}{r}0.026 \\
(0.011)\end{array}$ & $\begin{array}{r}-0.033 \\
(0.017)\end{array}$ & $\begin{array}{r}0.087 \\
(0.013)\end{array}$ \\
\hline
\end{tabular}

Note: ( $\mathrm{N}=9145$ for each row). Standard errors in parentheses. Standard errors for Trimming Proportion computed by the delta method. Bounds computed according to Table IV. See text for details. 
Appendix Table I: Summary Statistics, by Treatment Status, National Job Corps Study Conditional on Positive Earnings in Week 90

\begin{tabular}{|c|c|c|c|c|c|c|}
\hline \multirow[b]{2}{*}{ Variable } & \multicolumn{2}{|l|}{ Control } & \multicolumn{2}{|l|}{ Program } & \multicolumn{2}{|l|}{ Difference } \\
\hline & $\begin{array}{l}\text { Prop. Non- } \\
\text { Missing }\end{array}$ & Mean & $\begin{array}{l}\text { Prop. Non- } \\
\text { Missing }\end{array}$ & Mean & Diff. & Std. Err. \\
\hline Female & 1.00 & 0.429 & 1.00 & 0.419 & -0.009 & 0.016 \\
\hline Age at Baseline & 1.00 & 18.691 & 1.00 & 18.729 & 0.038 & 0.068 \\
\hline White, Non-Hispanic & 1.00 & 0.310 & 1.00 & 0.328 & 0.018 & 0.015 \\
\hline Black, Non-Hispanic & 1.00 & 0.447 & 1.00 & 0.443 & -0.004 & 0.016 \\
\hline Hispanic & 1.00 & 0.171 & 1.00 & 0.167 & -0.004 & 0.012 \\
\hline Other Race/Ethnicity & 1.00 & 0.072 & 1.00 & 0.063 & -0.009 & 0.008 \\
\hline Never married & 0.99 & 0.909 & 0.99 & 0.909 & 0.000 & 0.009 \\
\hline Married & 0.99 & 0.030 & 0.99 & 0.023 & -0.007 & 0.005 \\
\hline Living together & 0.99 & 0.039 & 0.99 & 0.045 & 0.006 & 0.006 \\
\hline Separated & 0.99 & 0.022 & 0.99 & 0.022 & 0.001 & 0.005 \\
\hline Has Child & 0.99 & 0.188 & 1.00 & 0.178 & -0.009 & 0.012 \\
\hline Number of children & 0.99 & 0.247 & 0.99 & 0.241 & -0.007 & 0.019 \\
\hline Education & 0.99 & 10.381 & 0.98 & 10.371 & -0.010 & 0.050 \\
\hline Mother's Educ. & 0.83 & 11.506 & 0.84 & 11.579 & 0.072 & 0.090 \\
\hline Father's Educ. & 0.66 & 11.644 & 0.67 & 11.458 & -0.186 & 0.111 \\
\hline Ever Arrested & 0.99 & 0.238 & 0.99 & 0.232 & -0.006 & 0.013 \\
\hline Household Inc: $<3000$ & 0.68 & 0.188 & 0.66 & 0.202 & 0.014 & 0.015 \\
\hline $3000-6000$ & 0.68 & 0.188 & 0.66 & 0.182 & -0.006 & 0.015 \\
\hline $6000-9000$ & 0.68 & 0.116 & 0.66 & 0.119 & 0.003 & 0.012 \\
\hline $9000-18000$ & 0.68 & 0.289 & 0.66 & 0.270 & -0.019 & 0.017 \\
\hline$>18000$ & 0.68 & 0.219 & 0.66 & 0.227 & 0.008 & 0.016 \\
\hline Personal Inc: $<3000$ & 0.95 & 0.726 & 0.93 & 0.732 & 0.005 & 0.014 \\
\hline $3000-6000$ & 0.95 & 0.164 & 0.93 & 0.154 & -0.010 & 0.012 \\
\hline $6000-9000$ & 0.95 & 0.065 & 0.93 & 0.068 & 0.003 & 0.008 \\
\hline$>9000$ & 0.95 & 0.045 & 0.93 & 0.047 & 0.002 & 0.007 \\
\hline At Baseline: & & & & & & \\
\hline Have Job & 0.98 & 0.251 & 0.98 & 0.254 & 0.002 & 0.014 \\
\hline Mos. Empl. Prev. Yr. & 1.00 & 4.572 & 1.00 & 4.558 & -0.013 & 0.143 \\
\hline Had Job, Prev. Yr. & 0.99 & 0.725 & 0.99 & 0.727 & 0.002 & 0.014 \\
\hline Earnings, Prev. Yr. & 0.94 & 3783.940 & 0.94 & 3699.524 & -84.416 & 159.333 \\
\hline Usual Hours/Week & 1.00 & 24.600 & 1.00 & 25.165 & 0.565 & 0.642 \\
\hline Usual Wkly Earnings & 1.00 & 125.147 & 1.00 & 126.297 & 1.150 & 3.838 \\
\hline $\begin{array}{l}\text { After Random Assignm } \\
\text { Week } 90 \ln \text { (wage) }\end{array}$ & 1.00 & 1.827 & 1.00 & 1.870 & $0.043 *$ & 0.011 \\
\hline Number of Obs & 1660 & & 2564 & & & \\
\hline
\end{tabular}

Note: $\mathrm{N}=4224$. * denotes difference is statistically significant from 0 at the 5 percent level. Computations use design weights. Chi-square test of all coefficients equalling zero, from a logit of the treatment indicator on all baseline characteristics (where mean values were imputed for missing values) yields 19.50; associated p-value from a chisquared (27 dof) distribution is 0.851 . 